\title{
Exogenous Gibberellic Acid or Dilute Bee Honey Boosts Drought Stress Tolerance in Vicia faba by Rebalancing Osmoprotectants, Antioxidants, Nutrients, and Phytohormones
}

\author{
Mostafa M. Rady ${ }^{1, *(1)}$, Sara H. K. Boriek ${ }^{1}$, Taia A. Abd El-Mageed ${ }^{2}$, Mohamed A. Seif El-Yazal ${ }^{1}$, Esmat F. Ali ${ }^{3}{ }^{1}$, \\ Fahmy A. S. Hassan ${ }^{3}$ (D) and Abdelsattar Abdelkhalik $4, *$ (D) \\ 1 Botany Department, Faculty of Agriculture, Fayoum University, Fayoum 63514, Egypt; \\ sarahamdy396@gmail.com (S.H.K.B.); maa04@fayoum.edu.eg (M.A.S.E.-Y.) \\ 2 Soil and Water Science Department, Faculty of Agriculture, Fayoum University, Fayoum 63514, Egypt; \\ taa00@fayoum.edu.eg \\ 3 Department of Biology, College of Science, Taif University, P.O. Box 11099, Taif 21944, Saudi Arabia; \\ a.esmat@tu.edu.sa (E.F.A.); d.fahmy@tu.edu.sa (F.A.S.H.) \\ 4 Horticulture Department, Faculty of Agriculture, Fayoum University, Fayoum 63514, Egypt \\ * Correspondence: mmr02@fayoum.edu.eg (M.M.R.); aga04@fayoum.edu.eg (A.A.); Tel.: +20-84-010-923-920-38
}

Citation: Rady, M.M.; Boriek, S.H.K.; Abd El-Mageed, T.A.; Seif El-Yazal, M.A.; Ali, E.F.; Hassan, F.A.S.; Abdelkhalik, A. Exogenous Gibberellic Acid or Dilute Bee Honey Boosts Drought Stress Tolerance in Vicia faba by Rebalancing Osmoprotectants, Antioxidants, Nutrients, and Phytohormones. Plants 2021, 10, 748. https://doi.org/ $10.3390 /$ plants10040748

Academic Editor: Anelia Dobrikova

Received: 20 March 2021

Accepted: 8 April 2021

Published: 11 April 2021

Publisher's Note: MDPI stays neutral with regard to jurisdictional claims in published maps and institutional affiliations.

Copyright: (C) 2021 by the authors Licensee MDPI, Basel, Switzerland. This article is an open access article distributed under the terms and conditions of the Creative Commons Attribution (CC BY) license (https:// creativecommons.org/licenses/by/ $4.0 /)$.
Abstract: The use of growth regulators such as gibberellic acid $\left(\mathrm{GA}_{3}\right)$ and biostimulants, including diluted bee honey $(\mathrm{Db}-\mathrm{H})$ can improve drought tolerance in many crops, including the faba bean (Vicia faba L.). Db-H contains high values of osmoprotectants, mineral nutrients, vitamins, and many antioxidants making it an effective growth regulator against environmental stress effects. Therefore, the present study was planned to investigate the potential improvement in the faba bean plant performance (growth and productivity) under full watering (100\% of crop evapotranspiration (ETc)) and drought stress $\left(60 \%\right.$ of ETc) by foliar application of $\mathrm{GA}_{3}\left(20 \mathrm{mg} \mathrm{L}^{-1}\right)$ or Db-H $\left(20 \mathrm{~g} \mathrm{~L}^{-1}\right)$. The ameliorative impacts of these growth regulators on growth, productivity, physio-biochemical attributes, nutrient status, antioxidant defense system, and phytohormones were evaluated. $\mathrm{GA}_{3}$ or $\mathrm{Db}-\mathrm{H}$ attenuated the negative influences of drought stress on cell membrane stability, ion leakage, relative water content, nutrient status, leaf pigments related to photosynthesis (chlorophylls and carotenoids), and efficiency of the photosystem II (PSII in terms of $F_{v} / F_{m}$ and performance index), thus improving faba bean growth, green pod yield, and water use efficiency. Drought stress caused an abnormal state of nutrients and photosynthetic machinery due to increased indicators of oxidative stress (malondialdehyde (MDA), hydrogen peroxide $\left(\mathrm{H}_{2} \mathrm{O}_{2}\right)$ and superoxide $\left(\mathrm{O}_{2}{ }^{\bullet-}\right)$ ), associated with increased osmoprotectants (proline, glycine betaine, soluble sugars, and soluble protein), nonenzymatic antioxidants (ascorbic acid, glutathione, and $\alpha$-tocopherol), and enzymatic antioxidant activities (superoxide dismutase, catalase, glutathione reductase, and ascorbate peroxidase). However, foliar-applied $\mathrm{GA}_{3}$ or $\mathrm{Db}-\mathrm{H}$ mediated further increases in osmoprotectants, antioxidant capacity, $\mathrm{GA}_{3}$, indole-3-acetic acid, and cytokinins, along with decreased levels of MDA and abscisic acid. These results suggest the use of $\mathrm{GA}_{3}$ or $\mathrm{Db}-\mathrm{H}$ at the tested concentrations to mitigate drought-induced damage in bean plants to obtain satisfactory growth and productivity under a water deficit of up to $40 \%$.

Keywords: faba bean; drought; growth and productivity; antioxidants defense system; biostimulants

\section{Introduction}

Among the most important legume crops, the faba bean (Vicia faba L.) is widely cultivated around the world. Fresh pods and dry seeds are consumed worldwide for humans due to their nutritional value, which is considered among vegetables [1]. Faba bean is rich in protein (up to 35\% of dry matter) [2], carbohydrates (51-68\% of dry matter) [1], and mineral nutrients such as potassium $(\mathrm{K})$, iron $(\mathrm{Fe})$, calcium $(\mathrm{Ca})$, magnesium $(\mathrm{Mg})$, and zinc $(\mathrm{Zn})[2,3]$. 
Limited irrigation water is one of the biggest limiting factors for crop production $[4,5]$, given that irrigated agriculture is the largest user of freshwater, with approximately $79 \%$ in Egypt and 69\% worldwide of total water withdrawals [6]. Dwindling freshwater resources along with meeting the demand for food production requires increased water use efficiency (WUE) in both irrigated and rainfed agriculture $[7,8]$.

Drought or water deficit directly impedes plant growth and productivity by causing loss of cell turgor and impairing mitosis that hinders cell elongation and division $[9,10]$. Osmotic stress is the primary signal in response to drought stress that induces abscisic acid (ABA) accumulation, which in turn, elicits several responses in plant cells [11,12]. As a secondary response, excessive formation of reactive oxygen species (ROS) such as hydroxyl radicals $\left(\mathrm{OH}^{-}\right)$, hydrogen peroxide $\left(\mathrm{H}_{2} \mathrm{O}_{2}\right)$, and superoxide radicals $\left(\mathrm{O}_{2}{ }^{\bullet-}\right)$ occurs due to drought in plant organelles like chloroplasts, mitochondria, and peroxisome $[13,14]$. These ROS disrupt the normal balance that exists between ROS production and scavenging [15]. This off-balance (due to excessive formation of ROS) not only inhibits the activity of various enzymes but also induces oxidative damage to cellular components such as DNA, protein, and lipids $[15,16]$. Concurrently, ROS affect cellular function and modulate stress-related primary and secondary metabolites and disturb redox homeostasis [9]. Moreover, ROS cause chlorophyll degradation and reduction of membrane stability [4,14]. A prolonged water deficit may cause cell death as a result of the massive production of ROS, which inhibits the scavenging action of the antioxidants machinery [17]. To prevent oxidative damage, plants have evolved adaptive mechanisms including upregulation of antioxidant defense system activity, which includes ROS-scavenger enzymes (e.g., ascorbate peroxidase (APX), catalase (CAT), glutathione reductase (GR), and superoxide dismutase (SOD)) and non-enzymatic antioxidants (e.g., glutathione, $\alpha$-tocopherol, ascorbic acid, and phenolic compounds) [18-20]. Moreover, the accumulation of osmoprotectants (e.g., glycine betaine, soluble sugars, and proline) contributes to the maintenance of cell turgor by means of osmotic adjustment $[4,21]$. Therefore, under drought stress, it is imperative to provide sustainable strategies to support plants to resist such stress.

Gibberellins $\left(\mathrm{GA}_{\mathrm{s}}\right)$ are phytohormones involved in plant growth and development; stem and root elongation, leaf expansion, flowering, and seed germination, as $\mathrm{GA}_{\mathrm{s}}$ regulate various metabolic processes, activity of various enzymes, and gene expression [22,23]. Based on previous observations, gibberellic acid $\left(\mathrm{GA}_{3}\right)$ plays a pivotal role in relieving abiotic stress [24-26]. Exogenous application of $\mathrm{GA}_{3}$ improves stomatal conductance, net photosynthesis rate, ion uptake, and hormonal balance [25]. Besides enhancing water use efficiency (WUE) [22,24], $\mathrm{GA}_{3}$ boosts antioxidant capacity [15], minimizes lipid peroxidation, and upregulates enzymatic antioxidants and osmoprotectants $[27,28]$ to mitigate the adverse influences of drought stress. $\mathrm{GA}_{\mathrm{S}}$ crosstalk with other phytohormones to regulate several metabolic processes during plant growth $[29,30]$. The biosynthesis of $\mathrm{GA}_{\mathrm{S}}$ is promoted by indole-3-acetic acid, while $\mathrm{GA}_{\mathrm{s}}$ catabolize ABA [25,29].

Biostimulants are a promising sustainable strategy to stimulate plant growth and productivity and to strengthen the plant's ability to mitigate abiotic stresses $[19,31,32]$. Although the use of commercially available plant growth stimulants such as osmoprotectants and/or antioxidants reduces the deleterious effects of abiotic stress, they are costly to growers. However, natural-based biostimulants such as plant-derived protein hydrolysate, Moringa oleifera leaves, propolis, maize grains, licorice roots, and diluted bee honey extracts are inexpensive by-products of plants or organisms that contribute to sustainable agriculture as an alternative to synthetic protectants [26,33-39]. The direct effect beyond the natural-based biostimulants is due to the fact that they contain many plant growth-promoting molecules such as antioxidants, osmoprotectants, mineral nutrients, and phytohormones. These growth-promoting molecules trigger physiological and biochemical changes, increase water and nutrient uptake, as well as promote resilience against abiotic stress including drought stress [31,36]. Diluted bee honey $(\mathrm{Db}-\mathrm{H})$ is a natural solution that mainly contains monosaccharides, disaccharides, and oligosaccharides [40,41]. Moreover, it contains various substances such as minerals, enzymes, proteins, lipids, or- 
ganic acids, inorganic acids, and phenolic compounds (phenolic acids, flavonoids) [41,42]. $\mathrm{Db}-\mathrm{H}$ serves as an active antioxidant in scavenging ROS [38,41] due to the presence of flavonoids that inhibit auto-oxidation [42] and enzymes that contribute to the removal of oxygen radicals [41], which are effective protection against drought-induced oxidative damage. As stated by Teklić et al. [32], Bulgari et al. [43], and Semida et al. [38], diluted honey extract as a plant biostimulator can increase tolerance to abiotic stress in plants. A recent field study highlighted the ability of Db-H-based plant biostimulants to alleviate salt stress in onions [38]. Indeed, Db-H applied to onion leaves showed higher biomass production, bulb yield, WUE, and leaf photosynthetic pigment contents. Moreover, $\mathrm{Db}-\mathrm{H}$ promoted both enzymatic and non-enzymatic antioxidants, membrane integrity, and water content in onion tissues under the influence of salt stress.

However, to our knowledge, exogenous applications with $\mathrm{Db}-\mathrm{H}$ as a natural biostimulant along with $\mathrm{GA}_{3}$ to plants grown under drought stress have not been studied before. Therefore, the current study was planned to evaluate the possibility of using some growth regulators; $\mathrm{Db}-\mathrm{H}$ or $\mathrm{GA}_{3}$ as a promising tool to relieve the adverse influences of water deficit stress on Vicia faba productivity. This research is designed to examine potential positive changes in physio-biochemical attributes, antioxidant defense system activity, and accumulation of osmoprotectants in faba bean plants growing under the influence of drought stress and foliar application of $\mathrm{Db}-\mathrm{H}$ or $\mathrm{GA}_{3}$. In this research, the potential improvement in plant growth, yield, WUE, and photosynthetic efficiency mediated by exogenous application of $\mathrm{Db}-\mathrm{H}$ or $\mathrm{GA}_{3}$ under drought stress conditions was also evaluated.

\section{Results}

\subsection{Growth and Green Pod Yield}

The results in Table 1 show that drought stress significantly decreased the growth traits of Vicia faba plants (leaf area plant ${ }^{-1}$, the number of leaves plant ${ }^{-1}$, and shoot dry weight plant $^{-1}$ ) by $22 \%$ and $23 \%, 26 \%$, and $25 \%$, and $41 \%$ and $43 \%$ in the $2018 / 2019$ and $2019 / 2020$ seasons, respectively, compared to the control. However, exogenously-applied $\mathrm{GA}_{3}$ or $\mathrm{Db}-\mathrm{H}$ notably increased all growth traits ( $\mathrm{Db}-\mathrm{H}$ recorded better enhancements) compared to the corresponding control. Foliar application of $\mathrm{GA} 3 \mathrm{or} \mathrm{Db}-\mathrm{H}$ to drought-stressed plants resulted in positive effects on faba bean growth characteristics and recorded identical values for plants grown under full irrigation without the use of any growth regulator $(100 \%$ of ETc). These effects of water deficit and foliar application of growth regulators on growth traits are reflected on the yield component. Irrigation of faba bean plants with $60 \%$ of ETc markedly decreased the green pods' number plant ${ }^{-1}$ by $30 \%$ and $29 \%$ and green pods yield by $48 \%$ and $45 \%$ in both seasons, respectively, compared to the control (100\% of ETc). However, exogenously-applied $\mathrm{GA}_{3}$ or $\mathrm{Db}-\mathrm{H}$ to faba bean plants compensated the yield reduction occurred through inducing substantial increases in the number of green pods per plant by $65 \%$ and $66 \%$ and green pods yield by 134 and $138 \%$ (seasons average) in the plants subjected to $60 \%$ of ETc, respectively, when compared with the corresponding control. It can be seen that the corrective action of $\mathrm{GA}_{3}$ and $\mathrm{Db}-\mathrm{H}$ can bring the pods yield achieved under drought stress to the same yield as achieved under optimum irrigation ( $100 \%$ of ETc). Under the tested irrigation regimes, WUE was differed, meaning that full irrigation recorded a WUE increase of $14 \%$ and $8 \%$ in both seasons, respectively, compared to the treatment of water deficit. The highest WUE corresponded with $100 \%$ of ETc $\times \mathrm{Db}-\mathrm{H}$ treatment, while the $60 \%$ of ETc $\times$ control treatment recorded the lowest WUE. However, foliar-applied $\mathrm{GA}_{3}$ and $\mathrm{Db}-\mathrm{H}$ to drought-stressed faba bean plants increased WUE by $63 \%$ and $66 \%$ (seasons average), respectively, compared to those obtained under fully irrigated plants that were not treated with any of the growth regulators (Table 1). 
Table 1. Foliar application of some growth regulators (e.g., gibberellic acid; GA 3 and diluted bee honey; Db-H) promoted growth and green pod yield components of Vicia faba plants grown under well watering (100\% of crop evapotranspiration; ETc) or deficit irrigation ( $60 \%$ of ETc).

\begin{tabular}{|c|c|c|c|c|c|c|}
\hline \multirow{2}{*}{$\begin{array}{l}\text { Source of } \\
\text { Variation }\end{array}$} & $\begin{array}{l}\text { No. of Leaves } \\
\text { per Plant }\end{array}$ & $\begin{array}{l}\text { Leaf Area per } \\
\text { Plant }\left(\mathrm{cm}^{2}\right)\end{array}$ & $\begin{array}{l}\text { Shoot DW per } \\
\text { Plant (g) }\end{array}$ & $\begin{array}{l}\text { No. of Green } \\
\text { Pods per Plant }\end{array}$ & $\begin{array}{l}\text { Green Pods Yield } \\
\text { per Hectare (ton) }\end{array}$ & $\begin{array}{l}\text { WUE }(\mathrm{Kg} \\
\left.\text { per } \mathrm{m}^{3}\right)\end{array}$ \\
\hline & \multicolumn{6}{|c|}{ Season of $2018 / 2019$} \\
\hline Irrigation (Ir) & $*$ & $*$ & $* *$ & $*$ & $* *$ & $*$ \\
\hline $100 \%$ of ETc & $31.8 \pm 3.1 \mathrm{a}$ & $136.0 \pm 13.7 \mathrm{a}$ & $17.7 \pm 1.7 \mathrm{a}$ & $20.2 \pm 1.7 \mathrm{a}$ & $32.5 \pm 2.9 a$ & $8.81 \pm 1.12 \mathrm{a}$ \\
\hline $60 \%$ of ETc & $24.8 \pm 2.4 b$ & $100.7 \pm 10.1 b$ & $10.4 \pm 1.0 \mathrm{~b}$ & $14.2 \pm 1.3 b$ & $16.8 \pm 1.7 \mathrm{~b}$ & $7.59 \pm 1.09 b$ \\
\hline Regulators (Re) & $*$ & $*$ & * & $*$ & $*$ & $*$ \\
\hline Control (Cn) & $23.5 \pm 2.3 c$ & $95.0 \pm 10.3 c$ & $10.1 \pm 1.0 \mathrm{c}$ & $13.3 \pm 1.3 c$ & $15.1 \pm 1.5 c$ & $5.12 \pm 0.88 c$ \\
\hline $\mathrm{GA}_{3}$ & $29.7 \pm 3.0 b$ & $125.8 \pm 12.6 b$ & $15.0 \pm 1.6 b$ & $18.2 \pm 1.4 b$ & $26.9 \pm 2.3 b$ & $9.11 \pm 1.03 b$ \\
\hline $\mathrm{Db}-\mathrm{H}$ & $31.7 \pm 3.0 \mathrm{a}$ & $134.3 \pm 12.9 \mathrm{a}$ & $17.2 \pm 1.6 \mathrm{a}$ & $20.0 \pm 1.9 a$ & $32.1 \pm 3.1 \mathrm{a}$ & $10.87 \pm 1.21 \mathrm{a}$ \\
\hline $\operatorname{Ir} \times \operatorname{Re}$ & * & $*$ & $*$ & $*$ & $*$ & $*$ \\
\hline $100 \%$ ETc $\times$ Cn & $28.3 \pm 2.7 c$ & $117.5 \pm 12.1 \mathrm{c}$ & $11.9 \pm 1.2 \mathrm{c}$ & $16.5 \pm 1.3 c$ & $20.8 \pm 1.8 \mathrm{c}$ & $5.64 \pm 0.98 d$ \\
\hline $100 \% \mathrm{ETc} \times \mathrm{GA}_{3}$ & $31.7 \pm 3.4 b$ & $137.7 \pm 14.2 b$ & $18.5 \pm 1.9 \mathrm{~b}$ & $20.3 \pm 1.5 b$ & $33.3 \pm 2.4 b$ & $9.02 \pm 1.13 b$ \\
\hline $\begin{array}{c}100 \% \text { ETc } \times \\
\text { Db-H }\end{array}$ & $35.3 \pm 3.2 \mathrm{a}$ & $152.7 \pm 14.8 \mathrm{a}$ & $22.7 \pm 2.1 \mathrm{a}$ & $23.7 \pm 2.4 \mathrm{a}$ & $43.5 \pm 4.4 \mathrm{a}$ & $11.79 \pm 1.23 \mathrm{a}$ \\
\hline $60 \%$ ETc $\times$ Cn & $18.7 \pm 1.9 c$ & $72.4 \pm 8.4 \mathrm{~d}$ & $8.2 \pm 0.7 d$ & $10.1 \pm 1.3 \mathrm{~d}$ & $9.4 \pm 1.1 d$ & $4.25 \pm 0.86 c$ \\
\hline $60 \%$ ETc $\times \mathrm{GA}_{3}$ & $27.7 \pm 2.6 c$ & $113.8 \pm 11.0 \mathrm{c}$ & $11.4 \pm 1.2 \mathrm{c}$ & $16.1 \pm 1.3 c$ & $20.4 \pm 2.1 c$ & $9.21 \pm 0.99 b$ \\
\hline \multirow[t]{2}{*}{$60 \%$ ETc $\times \mathrm{Db}-\mathrm{H}$} & $28.0 \pm 2.8 \mathrm{c}$ & $115.9 \pm 10.9 c$ & $11.7 \pm 1.0 \mathrm{c}$ & $16.3 \pm 1.4 \mathrm{c}$ & $20.7 \pm 1.8 \mathrm{c}$ & $9.35 \pm 1.21 b$ \\
\hline & \multicolumn{6}{|c|}{ Season of $2019 / 2020$} \\
\hline Irrigation (Ir) & $*$ & $*$ & $* *$ & $*$ & $* *$ & $*$ \\
\hline $100 \%$ of ETc & $33.1 \pm 3.2 \mathrm{a}$ & $152.4 \pm 13.3 a$ & $19.8 \pm 2.0 \mathrm{a}$ & $19.1 \pm 2.2 \mathrm{a}$ & $31.2 \pm 3.1 \mathrm{a}$ & $8.31 \pm 0.93 a$ \\
\hline $60 \%$ of ETc & $25.6 \pm 2.4 b$ & $113.9 \pm 11.1 b$ & $11.2 \pm 1.0 \mathrm{~b}$ & $13.5 \pm 1.5 b$ & $17.2 \pm 1.7 \mathrm{~b}$ & $7.64 \pm 1.02 b$ \\
\hline Regulators (Re) & $*$ & $*$ & $*$ & $*$ & $*$ & $*$ \\
\hline Control (Cn) & $24.2 \pm 2.4 b$ & $105.6 \pm 9.3 c$ & $10.7 \pm 1.1 c$ & $12.6 \pm 1.4 \mathrm{c}$ & $15.2 \pm 1.7 \mathrm{c}$ & $5.06 \pm 1.03 c$ \\
\hline $\mathrm{GA}_{3}$ & $31.0 \pm 3.0 \mathrm{a}$ & $140.8 \pm 12.3 b$ & $17.2 \pm 1.8 b$ & $17.3 \pm 2.0 \mathrm{~b}$ & $26.6 \pm 2.6 b$ & $8.86 \pm 1.16 b$ \\
\hline $\mathrm{Db}-\mathrm{H}$ & $33.0 \pm 3.2 \mathrm{a}$ & $151.4 \pm 15.1 \mathrm{a}$ & $18.7 \pm 1.7 \mathrm{a}$ & $19.2 \pm 2.2 \mathrm{a}$ & $30.9 \pm 3.1 \mathrm{a}$ & $10.29 \pm 1.32 \mathrm{a}$ \\
\hline $\operatorname{Ir} \times \operatorname{Re}$ & * & $*$ & $*$ & $*$ & $*$ & $*$ \\
\hline $100 \%$ ETc $\times$ Cn & $29.4 \pm 2.8 c$ & $134.1 \pm 10.4 c$ & $12.7 \pm 1.3 c$ & $15.9 \pm 1.8 \mathrm{c}$ & $21.8 \pm 2.3 c$ & $5.81 \pm 0.69 d$ \\
\hline $100 \% \mathrm{ETc} \times \mathrm{GA}_{3}$ & $33.1 \pm 3.2 b$ & $150.2 \pm 12.2 b$ & $21.9 \pm 2.2 b$ & $18.8 \pm 2.2 b$ & $31.7 \pm 2.8 b$ & $8.44 \pm 1.06 \mathrm{c}$ \\
\hline $\begin{array}{c}100 \% \text { ETc } \times \\
\text { Db-H }\end{array}$ & $36.7 \pm 3.7 \mathrm{a}$ & $169.4 \pm 17.4 a$ & $24.8 \pm 2.3 a$ & $22.7 \pm 2.5 a$ & $40.1 \pm 4.2 \mathrm{a}$ & $10.68 \pm 1.22 \mathrm{a}$ \\
\hline $60 \%$ ETc $\times$ Cn & $18.9 \pm 1.9 \mathrm{~d}$ & $77.1 \pm 8.2 \mathrm{~d}$ & $8.7 \pm 0.9 \mathrm{~d}$ & $9.2 \pm 1.0 \mathrm{~d}$ & $8.5 \pm 1.0 \mathrm{~d}$ & $3.77 \pm 0.63 e$ \\
\hline $60 \%$ ETc $\times \mathrm{GA}_{3}$ & $28.8 \pm 2.7 c$ & $131.4 \pm 12.4 \mathrm{c}$ & $12.4 \pm 1.2 \mathrm{c}$ & $15.7 \pm 1.7 \mathrm{c}$ & $21.4 \pm 2.3 c$ & $9.50 \pm 1.11 b$ \\
\hline $60 \% \mathrm{ETc} \times \mathrm{Db}-\mathrm{H}$ & $29.2 \pm 2.6 c$ & $133.3 \pm 12.7 \mathrm{c}$ & $12.6 \pm 1.0 \mathrm{c}$ & $15.7 \pm 1.8 \mathrm{c}$ & $21.7 \pm 1.9 \mathrm{c}$ & $9.63 \pm 1.13 b$ \\
\hline
\end{tabular}

** and * indicate respectively differences at $p \leq 0.05$ and $p \leq 0.01$ probability level. Means followed by the same letter in each column are not significantly different according to the LSD test $(p \leq 0.05)$.

\subsection{Efficiency of the Photosynthetic Machinery}

As displayed in Table 2, water deficit (660\% of ETc) caused a considerable decrease in the leaf photosynthetic pigments (total chlorophylls and carotenoids contents), photochemical activity, SPAD chlorophyll index (soil-plant analysis development) values, and photosynthetic efficiency $\left(\mathrm{F}_{\mathrm{v}} / \mathrm{F}_{\mathrm{m}}\right.$ and performance index; PI) compared to full irrigation $(100 \%$ of ETc).

Compared to untreated control plants, sprayed plants with $\mathrm{GA}_{3}$ or $\mathrm{Db}-\mathrm{H}$ showed higher photosynthetic pigment contents, SPAD chlorophyll index, photochemical activity, and the efficiency of PSII. In fully irrigated plants, application of $\mathrm{GA}_{3}$ or $\mathrm{Db}-\mathrm{H}$ increased total chlorophylls by $15 \%$ and $24 \%$, total carotenoids by $10 \%$ and $18 \%$, photochemical activity by $8 \%$ and $13 \%$, SPAD index by $8 \%$ and $14 \%, F_{v} / F_{m}$ by $6 \%$ and $11 \%$, and performance index by $7 \%$ and $20 \%$ (seasons average), respectively, in comparison to the corresponding control. Foliage-applied $\mathrm{GA}_{3}$ or $\mathrm{Db}-\mathrm{H}$ alleviated the negative effects on the photosynthetic machinery in drought-stressed faba bean plants. In deficit-irrigated plants, the increases in the photosynthetic machinery (total chlorophylls, total carotenoids, photochemical activity, SPAD chlorophyll index, $\left.\mathrm{F}_{\mathrm{v}} / \mathrm{F}_{\mathrm{m}}, \mathrm{PI}\right)$ were $59 \%$ and $62 \%, 27 \%$ and $31 \%, 32 \%$ and $32 \%, 40 \%$ and $41 \%, 18 \%$ and $21 \%$, and $52 \%$ and $53 \%$ (seasons average), respectively, compared with the corresponding control. 
Table 2. Foliar application of some growth regulators (e.g., gibberellic acid; $\mathrm{GA}_{3}$ and diluted bee honey; $\mathrm{Db}-\mathrm{H}$ ) promoted photosynthetic machinery efficiency of Vicia faba plants grown under well watering (100\% of crop evapotranspiration; ETc) or deficit irrigation $(60 \%$ of ETc).

\begin{tabular}{|c|c|c|c|c|c|c|}
\hline \multirow{2}{*}{$\begin{array}{l}\text { Source of } \\
\text { Variation }\end{array}$} & $\begin{array}{l}\text { Total Chlorophylls } \\
\text { (mg per g FW) }\end{array}$ & $\begin{array}{l}\text { Total Carotenoids } \\
(\mathrm{mg} \text { per } \mathrm{g} \text { FW) }\end{array}$ & $\begin{array}{c}\text { Photochemical } \\
\text { Activity }\end{array}$ & $\begin{array}{l}\text { SPAD Chlorophyll } \\
\text { Index }\end{array}$ & $F_{v} / F_{m}$ & $\begin{array}{l}\text { Performance } \\
\text { Index }(\%)\end{array}$ \\
\hline & \multicolumn{6}{|c|}{ Season of $2018 / 2019$} \\
\hline Irrigation (Ir) & $*$ & $*$ & $*$ & $*$ & * & $*$ \\
\hline $100 \%$ of ETc & $3.13 \pm 0.19 a$ & $0.75 \pm 0.02 a$ & $45.2 \pm 1.6 a$ & $66.6 \pm 2.4 a$ & $0.85 \pm 0.02 a$ & $16.8 \pm 0.21 \mathrm{a}$ \\
\hline $60 \%$ of ETc & $2.44 \pm 0.12 b$ & $0.63 \pm 0.01 b$ & $38.9 \pm 1.3 b$ & $56.8 \pm 1.9 b$ & $0.77 \pm 0.02 b$ & $13.8 \pm 0.17 \mathrm{~b}$ \\
\hline Regulators (Re) & $*$ & $*$ & $*$ & $*$ & $*$ & $*$ \\
\hline Control (Cn) & $2.33 \pm 0.12 c$ & $0.60 \pm 0.01 c$ & $37.5 \pm 1.2 \mathrm{~b}$ & $54.0 \pm 1.8 b$ & $0.75 \pm 0.02 b$ & $13.0 \pm 0.18 c$ \\
\hline $\mathrm{GA}_{3}$ & $2.94 \pm 0.19 b$ & $0.72 \pm 0.02 b$ & $43.7 \pm 1.6 \mathrm{a}$ & $64.7 \pm 2.5 a$ & $0.83 \pm 0.03 a$ & $16.0 \pm 0.19 b$ \\
\hline $\mathrm{Db}-\mathrm{H}$ & $3.10 \pm 0.15 a$ & $0.76 \pm 0.02 a$ & $45.0 \pm 1.7 \mathrm{a}$ & $66.4 \pm 2.2 c$ & $0.86 \pm 0.03 a$ & $17.1 \pm 0.22 \mathrm{a}$ \\
\hline $\operatorname{Ir} \times \operatorname{Re}$ & $*$ & $*$ & $*$ & $*$ & $*$ & $*$ \\
\hline $100 \% \mathrm{ETc} \times \mathrm{Cn}$ & $2.78 \pm 0.17 \mathrm{c}$ & $0.68 \pm 0.01 c$ & $42.3 \pm 1.3 b$ & $62.4 \pm 2.1 c$ & $0.81 \pm 0.02 b$ & $15.8 \pm 0.21 b$ \\
\hline $100 \% \mathrm{ETc} \times \mathrm{GA}_{3}$ & $3.18 \pm 0.21 b$ & $0.76 \pm 0.02 b$ & $45.4 \pm 1.6 a$ & $67.1 \pm 2.7 \mathrm{a}$ & $0.85 \pm 0.02 \mathrm{ab}$ & $16.3 \pm 0.18 b$ \\
\hline $100 \%$ ETc $\times$ Db-H & $3.42 \pm 0.18 \mathrm{a}$ & $0.82 \pm 0.02 a$ & $47.9 \pm 2.0 \mathrm{a}$ & $70.2 \pm 2.4 a$ & $0.89 \pm 0.03 a$ & $18.4 \pm 0.25 a$ \\
\hline $60 \%$ ETc $\times$ Cn & $1.87 \pm 0.07 \mathrm{~d}$ & $0.52 \pm 0.00 \mathrm{~d}$ & $32.6 \pm 1.1 c$ & $45.6 \pm 1.4 \mathrm{~d}$ & $0.69 \pm 0.01 c$ & $10.1 \pm 0.14 c$ \\
\hline $60 \% \mathrm{ETc} \times \mathrm{GA}_{3}$ & $2.69 \pm 0.16 c$ & $0.68 \pm 0.01 c$ & $41.9 \pm 1.5 b$ & $62.3 \pm 2.2 c$ & $0.80 \pm 0.03 b$ & $15.6 \pm 0.20 \mathrm{~b}$ \\
\hline \multirow[t]{2}{*}{$60 \%$ ETc $\times$ Db-H } & $2.77 \pm 0.12 c$ & $0.70 \pm 0.02 c$ & $42.1 \pm 1.3 b$ & $62.5 \pm 2.0 c$ & $0.82 \pm 0.02 b$ & $15.7 \pm 0.18 b$ \\
\hline & \multicolumn{6}{|c|}{ Season of $2019 / 2020$} \\
\hline Irrigation (Ir) & $*$ & $*$ & $*$ & $*$ & $*$ & $*$ \\
\hline $100 \%$ of ETc & $3.41 \pm 0.14 \mathrm{a}$ & $0.76 \pm 0.03 a$ & $46.1 \pm 1.6 \mathrm{a}$ & $68.2 \pm 2.2 \mathrm{a}$ & $0.85 \pm 0.03 a$ & $17.2 \pm 0.17 \mathrm{a}$ \\
\hline $60 \%$ of ETc & $2.60 \pm 0.11 b$ & $0.65 \pm 0.01 b$ & $39.2 \pm 1.5 b$ & $57.1 \pm 2.0 b$ & $0.75 \pm 0.02 b$ & $13.7 \pm 0.13 b$ \\
\hline Regulators (Re) & $*$ & $*$ & $*$ & $*$ & $*$ & $*$ \\
\hline Control (Cn) & $2.38 \pm 0.10 b$ & $0.63 \pm 0.01 b$ & $37.5 \pm 1.5 b$ & $53.9 \pm 2.1 b$ & $0.73 \pm 0.02 b$ & $12.9 \pm 0.14 b$ \\
\hline $\mathrm{GA}_{3}$ & $3.26 \pm 0.13 a$ & $0.73 \pm 0.02 a$ & $44.6 \pm 1.8 \mathrm{a}$ & $65.7 \pm 2.0 \mathrm{a}$ & $0.83 \pm 0.02 \mathrm{a}$ & $16.3 \pm 0.16 a$ \\
\hline $\mathrm{Db}-\mathrm{H}$ & $3.40 \pm 0.14 \mathrm{a}$ & $0.76 \pm 0.03 a$ & $46.0 \pm 1.6 \mathrm{a}$ & $68.3 \pm 2.3 a$ & $0.85 \pm 0.04 \mathrm{a}$ & $17.2 \pm 0.16 \mathrm{a}$ \\
\hline $\operatorname{Ir} \times \operatorname{Re}$ & $*$ & $*$ & $*$ & $*$ & $*$ & $*$ \\
\hline $100 \% \mathrm{ETc} \times \mathrm{Cn}$ & $3.01 \pm 0.12 b$ & $0.70 \pm 0.02 b$ & $43.1 \pm 1.5 b$ & $63.5 \pm 2.4 b$ & $0.80 \pm 0.03 b$ & $15.5 \pm 0.16 c$ \\
\hline $100 \% \mathrm{ETc} \times \mathrm{GA}_{3}$ & $3.49 \pm 0.15 a$ & $0.76 \pm 0.02 \mathrm{a}$ & $46.4 \pm 1.8 \mathrm{a}$ & $68.1 \pm 1.9 \mathrm{a}$ & $0.86 \pm 0.02 \mathrm{a}$ & $17.1 \pm 0.18 b$ \\
\hline $100 \%$ ETc $\times$ Db-H & $3.74 \pm 0.14 \mathrm{a}$ & $0.81 \pm 0.04 a$ & $48.9 \pm 1.6 \mathrm{a}$ & $72.9 \pm 2.2 \mathrm{a}$ & $0.89 \pm 0.04 \mathrm{a}$ & $19.0 \pm 0.18 \mathrm{a}$ \\
\hline $60 \% \mathrm{ETc} \times \mathrm{Cn}$ & $1.74 \pm 0.08 c$ & $0.56 \pm 0.00 c$ & $31.8 \pm 1.4 c$ & $44.3 \pm 1.7 \mathrm{c}$ & $0.66 \pm 0.01 c$ & $10.3 \pm 0.11 d$ \\
\hline $60 \%$ ETc $\times \mathrm{GA}_{3}$ & $3.02 \pm 0.11 b$ & $0.69 \pm 0.02 b$ & $42.8 \pm 1.7 \mathrm{~b}$ & $63.3 \pm 2.1 b$ & $0.79 \pm 0.02 b$ & $15.4 \pm 0.14 c$ \\
\hline $60 \%$ ETc $\times$ Db-H & $3.05 \pm 0.14 b$ & $0.71 \pm 0.02 b$ & $43.0 \pm 1.5 b$ & $63.6 \pm 2.3 b$ & $0.80 \pm 0.04 b$ & $15.4 \pm 0.14 c$ \\
\hline
\end{tabular}

** and * indicate respectively differences at $p \leq 0.05$ and $p \leq 0.01$ probability level. Means followed by the same letter in each column are not significantly different according to the LSD test $(p \leq 0.05)$.

\subsection{Leaf Tissue Stability and Oxidative Stress Indicators}

Faba bean leaf tissue stability was assayed as the membrane stability index (MSI), electrolyte leakage (EL), and relative water content (RWC) (Table 3). For irrigation levels, the adverse effects of drought-induced stress on Vicia faba plants were described as decreases in RWC and MSI by 16\% and 20\%, while EL increased by $75 \%$ (seasons average), respectively, compared to irrigation with $100 \%$ of ETc. Regarding the foliar application of growth regulators, application of $\mathrm{GA}_{3}$ or Db-H elevated both RWC and MSI, while minimized EL compared to untreated plants (control). However, $\mathrm{GA}_{3}$ or $\mathrm{Db}-\mathrm{H}$ supplementation markedly attenuated the drought-induced damage to tissue stability in faba bean plants, as the same RWC, MSI, and EL values were recorded for well-watered plants that were not treated with any of the growth regulators.

The utility of the oxidative damage indicators identified in this study was lipid peroxidation, expressed in malondialdehyde (MDA) content, hydrogen peroxide $\left(\mathrm{H}_{2} \mathrm{O}_{2}\right)$, and superoxide $\left(\mathrm{O}_{2}{ }^{\bullet-}\right)$ contents (Table 3). For irrigation level, when irrigation level decreased from $100 \%$ to $60 \%$ of ETc, $\mathrm{MDA}, \mathrm{H}_{2} \mathrm{O}_{2}$, and $\mathrm{O}_{2}{ }^{--}$contents increased by $67 \%, 58 \%$, and $102 \%$, and $67 \%, 60 \%$, and $100 \%$ in both seasons, respectively. Regarding the growth regulator applications, $\mathrm{GA}_{3}$ or Db-H significantly decreased levels of $\mathrm{MDA}, \mathrm{H}_{2} \mathrm{O}_{2}$, and $\mathrm{O}_{2}{ }^{\bullet-}$ compared to the control. For integrative treatments under full irrigation, the best treatments were $100 \%$ of ETc $\times \mathrm{GA}_{3}$ or $\mathrm{Db}-\mathrm{H}$ which significantly decreased the oxidative stress biomarkers. Under water deficit ( $60 \%$ of ETc), the best treatment was $60 \%$ of ETc $\times \mathrm{GA}_{3}$ or Db-H, which significantly reduced $\mathrm{MDA}, \mathrm{H}_{2} \mathrm{O}_{2}$, and $\mathrm{O}_{2}{ }^{\bullet-}$ contents by $64 \%$ and $69 \%, 52 \%$ and $54 \%$, and 
$69 \%$ and $69 \%$ (seasons average), respectively, compared to the corresponding control (60\% of ETc).

Table 3. Foliar application of some growth regulators (e.g., gibberellic acid; $\mathrm{GA}_{3}$ and diluted bee honey; $\left.\mathrm{Db}-\mathrm{H}\right)$ promoted leaf tissue stability and levels of oxidative stress biomarkers in Vicia faba plants grown under well watering (100\% of crop evapotranspiration; ETc) or deficit irrigation (60\% of ETc).

\begin{tabular}{|c|c|c|c|c|c|c|}
\hline $\begin{array}{l}\text { Source of } \\
\text { Variation }\end{array}$ & $\begin{array}{c}\text { Relative Water } \\
\text { Content (\%) }\end{array}$ & $\begin{array}{c}\text { Membrane } \\
\text { Stability Index } \\
(\%)\end{array}$ & $\begin{array}{c}\text { Electrolyte } \\
\text { Leakage (\%) }\end{array}$ & $\begin{array}{l}\text { Malondialdehyde } \\
\text { Level ( } \mu \text { mole } \\
\text { per g FW) }\end{array}$ & $\begin{array}{c}\text { Hydrogen } \\
\text { Peroxide }\left(\mathrm{H}_{2} \mathrm{O}_{2}\right) \\
\text { Level }(\mu \mathrm{mole} \\
\text { per g FW) }\end{array}$ & $\begin{array}{c}\begin{array}{c}\text { Superoxide } \\
\left(\mathrm{O}_{2}^{\bullet-}\right) \text { Level } \\
(\mu \text { mole per } g \text { FW })\end{array}\end{array}$ \\
\hline & \multicolumn{6}{|c|}{ Season of $2018 / 2019$} \\
\hline Irrigation (Ir) & $*$ & $*$ & $* *$ & $* *$ & $* *$ & $* *$ \\
\hline $100 \%$ of ETc & $87.6 \pm 4.6 \mathrm{a}$ & $76.3 \pm 3.8 \mathrm{a}$ & $10.6 \pm 0.5 b$ & $0.12 \pm 0.01 b$ & $1.29 \pm 0.03 b$ & $0.50 \pm 0.01 b$ \\
\hline $60 \%$ of ETc & $74.0 \pm 4.3 b$ & $61.2 \pm 3.3 b$ & $18.9 \pm 1.0 \mathrm{a}$ & $0.20 \pm 0.01 \mathrm{a}$ & $2.04 \pm 0.02 \mathrm{a}$ & $1.01 \pm 0.02 \mathrm{a}$ \\
\hline Regulators (Re) & $*$ & $*$ & $*$ & $*$ & $*$ & $*$ \\
\hline Control (Cn) & $70.5 \pm 3.8 b$ & $55.6 \pm 3.3 b$ & $22.4 \pm 1.3 \mathrm{a}$ & $0.24 \pm 0.02 \mathrm{a}$ & $2.29 \pm 0.05 a$ & $1.22 \pm 0.03 a$ \\
\hline $\mathrm{GA}_{3}$ & $85.2 \pm 4.7 \mathrm{a}$ & $75.1 \pm 3.8 \mathrm{a}$ & $11.0 \pm 0.6 b$ & $0.13 \pm 0.01 b$ & $1.37 \pm 0.02 b$ & $0.53 \pm 0.02 b$ \\
\hline $\mathrm{Db}-\mathrm{H}$ & $86.8 \pm 4.9 \mathrm{a}$ & $75.5 \pm 3.6 \mathrm{a}$ & $10.8 \pm 0.5 b$ & $0.12 \pm 0.00 b$ & $1.34 \pm 0.02 b$ & $0.52 \pm 0.01 b$ \\
\hline $\operatorname{Ir} \times \operatorname{Re}$ & $*$ & $*$ & $*$ & $*$ & $*$ & $*$ \\
\hline $100 \%$ ETc $\times$ Cn & $82.6 \pm 4.5 b$ & $72.8 \pm 3.3 b$ & $11.1 \pm 0.6 b$ & $0.13 \pm 0.01 b$ & $1.46 \pm 0.04 b$ & $0.55 \pm 0.02 b$ \\
\hline $100 \% \mathrm{ETc} \times \mathrm{GA}_{3}$ & $88.9 \pm 4.2 \mathrm{a}$ & $77.9 \pm 4.1 \mathrm{a}$ & $10.4 \pm 0.6 b$ & $0.12 \pm 0.01 b$ & $1.21 \pm 0.02 \mathrm{c}$ & $0.48 \pm 0.01 c$ \\
\hline $100 \%$ ETc $\times$ Db-H & $91.4 \pm 5.1 \mathrm{a}$ & $78.1 \pm 3.9 a$ & $10.2 \pm 0.4 b$ & $0.12 \pm 0.00 b$ & $1.19 \pm 0.02 c$ & $0.47 \pm 0.01 c$ \\
\hline $60 \% \mathrm{ETc} \times \mathrm{Cn}$ & $58.3 \pm 3.0 c$ & $38.4 \pm 3.2 \mathrm{c}$ & $33.7 \pm 2.0 \mathrm{a}$ & $0.34 \pm 0.02 \mathrm{a}$ & $3.11 \pm 0.06 a$ & $1.88 \pm 0.04 a$ \\
\hline $60 \% \mathrm{ETc} \times \mathrm{GA}_{3}$ & $81.4 \pm 5.1 b$ & $72.2 \pm 3.4 b$ & $11.6 \pm 0.5 b$ & $0.14 \pm 0.01 b$ & $1.52 \pm 0.02 b$ & $0.57 \pm 0.02 b$ \\
\hline \multirow[t]{2}{*}{$60 \%$ ETc $\times$ Db-H } & $82.2 \pm 4.7 \mathrm{~b}$ & $72.9 \pm 3.3 b$ & $11.3 \pm 0.6 b$ & $0.12 \pm 0.00 b$ & $1.48 \pm 0.02 b$ & $0.57 \pm 0.01 b$ \\
\hline & \multicolumn{6}{|c|}{ Season of $2019 / 2020$} \\
\hline Irrigation (Ir) & $*$ & $*$ & $* *$ & $* *$ & $* *$ & $* *$ \\
\hline $100 \%$ of ETc & $88.3 \pm 5.1 \mathrm{a}$ & $76.2 \pm 3.7 \mathrm{a}$ & $10.4 \pm 0.4 b$ & $0.12 \pm 0.00 b$ & $1.36 \pm 0.09 b$ & $0.44 \pm 0.02 \mathrm{a}$ \\
\hline $60 \%$ of ETc & $74.5 \pm 4.0 \mathrm{~b}$ & $61.0 \pm 3.9 b$ & $17.8 \pm 0.7 a$ & $0.20 \pm 0.01 \mathrm{a}$ & $2.18 \pm 0.11 \mathrm{a}$ & $0.88 \pm 0.04 a$ \\
\hline Regulators (Re) & $*$ & $*$ & * & $*$ & $*$ & $*$ \\
\hline Control (Cn) & $70.5 \pm 4.3 b$ & $55.8 \pm 4.0 \mathrm{~b}$ & $21.5 \pm 0.9 a$ & $0.27 \pm 0.02 \mathrm{a}$ & $2.48 \pm 0.15 a$ & $1.06 \pm 0.06 \mathrm{a}$ \\
\hline $\mathrm{GA}_{3}$ & $86.1 \pm 4.7 \mathrm{a}$ & $74.8 \pm 4.0 \mathrm{a}$ & $10.6 \pm 0.5 b$ & $0.12 \pm 0.01 b$ & $1.45 \pm 0.10 \mathrm{~b}$ & $0.47 \pm 0.02 b$ \\
\hline $\mathrm{Db}-\mathrm{H}$ & $87.7 \pm 4.8 \mathrm{a}$ & $75.3 \pm 3.5 a$ & $10.4 \pm 0.3 b$ & $0.11 \pm 0.00 \mathrm{~b}$ & $1.37 \pm 0.06 b$ & $0.46 \pm 0.02 b$ \\
\hline $\operatorname{Ir} \times \operatorname{Re}$ & $*$ & $*$ & * & $*$ & $*$ & $*$ \\
\hline $100 \%$ ETc $\times$ Cn & $83.7 \pm 5.1 b$ & $71.4 \pm 3.3 b$ & $10.8 \pm 0.3 b$ & $0.15 \pm 0.01 b$ & $1.55 \pm 0.08 b$ & $0.49 \pm 0.03 b$ \\
\hline $100 \% \mathrm{ETc} \times \mathrm{GA}_{3}$ & $89.4 \pm 4.8 \mathrm{a}$ & $78.2 \pm 4.1 \mathrm{a}$ & $10.4 \pm 0.5 b$ & $0.11 \pm 0.00 \mathrm{~cd}$ & $1.30 \pm 0.12 c$ & $0.42 \pm 0.02 c$ \\
\hline $100 \% \mathrm{ETc} \times \mathrm{Db}-\mathrm{H}$ & $91.8 \pm 5.4 \mathrm{a}$ & $78.9 \pm 3.8 \mathrm{a}$ & $10.1 \pm 0.3 b$ & $0.11 \pm 0.00 \mathrm{~cd}$ & $1.22 \pm 0.07 c$ & $0.41 \pm 0.02 c$ \\
\hline $60 \% \mathrm{ETc} \times \mathrm{Cn}$ & $57.2 \pm 3.4 c$ & $40.1 \pm 4.6 c$ & $32.1 \pm 1.4 \mathrm{a}$ & $0.38 \pm 0.02 \mathrm{a}$ & $3.41 \pm 0.21 \mathrm{a}$ & $1.62 \pm 0.09 \mathrm{a}$ \\
\hline $60 \% \mathrm{ETc} \times \mathrm{GA}_{3}$ & $82.8 \pm 4.6 b$ & $71.3 \pm 3.8 b$ & $10.7 \pm 0.5 b$ & $0.12 \pm 0.01 c$ & $1.60 \pm 0.07 b$ & $0.52 \pm 0.02 b$ \\
\hline $60 \%$ ETc $\times$ Db-H & $83.6 \pm 4.1 b$ & $71.6 \pm 3.2 b$ & $10.7 \pm 0.3 b$ & $0.10 \pm 0.00 \mathrm{~d}$ & $1.52 \pm 0.05 b$ & $0.50 \pm 0.01 b$ \\
\hline
\end{tabular}

** and * indicate respectively differences at $p \leq 0.05$ and $p \leq 0.01$ probability level. Means followed by the same letter in each column are not significantly different according to the LSD test $(p \leq 0.05)$.

\subsection{Osmoprotectant Compounds}

Results of Table 4 display the contents of the osmoprotectants in terms of soluble sugars, free proline, glycine betaine, and total soluble protein, which increased significantly by $43 \%, 64 \%, 85 \%$, and $21 \%$ (seasons average) in drought-stressed plants. Nevertheless, under different irrigation regimes, the application of $\mathrm{GA}_{3}$ or $\mathrm{Db}-\mathrm{H}$ increased the contents of soluble sugars, free proline, and glycine betaine contents, while the total soluble protein content was decreased. Under optimum irrigation (100\% of ETc), the increases were $43 \%$ and $74 \%, 31 \%$ and $31 \%$, and $38 \%$ and $38 \%$ (seasons average), respectively, compared with the respective control. For osmotically-stressed plants sprayed with $\mathrm{GA}_{3}$ or $\mathrm{Db}-\mathrm{H}$, the elevations in the soluble sugars, free proline, and glycine betaine contents were $13 \%$ and $27 \%, 21 \%$ and $23 \%$, and $30 \%$ and $32 \%$ (seasons average), respectively in comparison to the corresponding control. 
Table 4. Foliar application of some growth regulators (e.g., gibberellic acid; $\mathrm{GA}_{3}$ and diluted bee honey; $\mathrm{Db}-\mathrm{H}$ ) promoted osmoprotectant contents of Vicia faba plants grown under well watering (100\% of crop evapotranspiration; ETc) or deficit irrigation $(60 \%$ of ETc).

\begin{tabular}{|c|c|c|c|c|}
\hline \multirow[t]{2}{*}{ Source of Variation } & $\begin{array}{l}\text { Soluble Sugars } \\
\text { (mg per g DW) }\end{array}$ & $\begin{array}{l}\text { Free Proline } \\
(\mu \mathrm{M} \text { per } \mathrm{g} W)\end{array}$ & $\begin{array}{l}\text { Glycine Betaine } \\
(\mu \mathrm{M} \text { per } \mathrm{g} \mathrm{DW})\end{array}$ & $\begin{array}{l}\text { Total Soluble Protein } \\
\text { (mg per g DW) }\end{array}$ \\
\hline & \multicolumn{4}{|c|}{ Season of $2018 / 2019$} \\
\hline Irrigation (Ir) & $*$ & $* *$ & $* *$ & * \\
\hline $100 \%$ of ETc & $14.1 \pm 0.3 b$ & $138.5 \pm 1.8 \mathrm{~b}$ & $22.4 \pm 0.4 b$ & $72.1 \pm 1.5 b$ \\
\hline $60 \%$ of ETc & $19.9 \pm 0.4 \mathrm{a}$ & $221.5 \pm 2.5 a$ & $41.4 \pm 0.7 \mathrm{a}$ & $88.6 \pm 1.9 a$ \\
\hline Regulators (Re) & $*$ & * & $*$ & $*$ \\
\hline Control (Cn) & $14.0 \pm 0.3 c$ & $154.4 \pm 1.6 b$ & $26.6 \pm 0.5 b$ & $85.4 \pm 1.8 \mathrm{a}$ \\
\hline $\mathrm{GA}_{3}$ & $17.1 \pm 0.4 b$ & $192.2 \pm 2.6 \mathrm{a}$ & $34.3 \pm 0.6 \mathrm{a}$ & $78.1 \pm 1.8 b$ \\
\hline $\mathrm{Db}-\mathrm{H}$ & $20.1 \pm 0.5 \mathrm{a}$ & $193.5 \pm 2.3 a$ & $34.9 \pm 0.7 \mathrm{a}$ & $77.6 \pm 1.5 b$ \\
\hline $\operatorname{Ir} \times \operatorname{Re}$ & * & $*$ & * & $*$ \\
\hline $100 \% \mathrm{ETc} \times \mathrm{Cn}$ & $10.4 \pm 0.2 \mathrm{e}$ & $114.2 \pm 1.5 \mathrm{~d}$ & $18.1 \pm 0.3 \mathrm{~d}$ & $71.8 \pm 1.5 c$ \\
\hline $100 \% \mathrm{ETc} \times \mathrm{GA}_{3}$ & $14.2 \pm 0.4 \mathrm{~d}$ & $149.3 \pm 2.0 c$ & $24.3 \pm 0.4 \mathrm{c}$ & $72.0 \pm 1.7 \mathrm{c}$ \\
\hline $100 \%$ ETc $\times$ Db-H & $17.8 \pm 0.4 \mathrm{c}$ & $152.1 \pm 1.8 \mathrm{c}$ & $24.8 \pm 0.6 c$ & $72.4 \pm 1.3 c$ \\
\hline $60 \%$ ETc $\times$ Cn & $17.5 \pm 0.3 c$ & $194.6 \pm 1.7 \mathrm{~b}$ & $35.1 \pm 0.6 b$ & $98.9 \pm 2.0 \mathrm{a}$ \\
\hline $60 \% \mathrm{ETc} \times \mathrm{GA}_{3}$ & $19.9 \pm 0.4 b$ & $235.1 \pm 3.1 \mathrm{a}$ & $44.2 \pm 0.7 \mathrm{a}$ & $84.2 \pm 1.9 b$ \\
\hline \multirow[t]{2}{*}{$60 \%$ ETc $\times$ Db-H } & $22.3 \pm 0.5 a$ & $234.9 \pm 2.8 \mathrm{a}$ & $45.0 \pm 0.7 \mathrm{a}$ & $82.7 \pm 1.7 \mathrm{~b}$ \\
\hline & \multicolumn{4}{|c|}{ Season of $2019 / 2020$} \\
\hline Irrigation (Ir) & * & $* *$ & $* *$ & $* *$ \\
\hline $100 \%$ of ETc & $17.2 \pm 0.4 b$ & $145.5 \pm 2.2 b$ & $20.8 \pm 0.3 b$ & $73.9 \pm 1.6 b$ \\
\hline $60 \%$ of ETc & $24.8 \pm 0.5 \mathrm{a}$ & $245.5 \pm 3.2 \mathrm{a}$ & $38.5 \pm 0.6 a$ & $87.6 \pm 1.8 \mathrm{a}$ \\
\hline Regulators (Re) & $*$ & $*$ & $*$ & $*$ \\
\hline Control (Cn) & $17.1 \pm 0.4 \mathrm{c}$ & $167.0 \pm 2.5 b$ & $23.9 \pm 0.4 b$ & $85.6 \pm 1.8 \mathrm{a}$ \\
\hline $\mathrm{GA}_{3}$ & $21.3 \pm 0.5 b$ & $209.0 \pm 3.0 \mathrm{a}$ & $32.5 \pm 0.5 a$ & $78.6 \pm 1.7 \mathrm{~b}$ \\
\hline $\mathrm{Db}-\mathrm{H}$ & $24.6 \pm 0.6 \mathrm{a}$ & $210.6 \pm 2.7 a$ & $32.6 \pm 0.5 a$ & $78.1 \pm 1.6 b$ \\
\hline $\operatorname{Ir} \times \operatorname{Re}$ & $*$ & $*$ & $*$ & * \\
\hline $100 \% \mathrm{ETc} \times \mathrm{Cn}$ & $12.1 \pm 0.3 e$ & $121.4 \pm 2.0 \mathrm{~d}$ & $16.4 \pm 0.2 \mathrm{~d}$ & $73.5 \pm 1.7 \mathrm{c}$ \\
\hline $100 \% \mathrm{ETc} \times \mathrm{GA}_{3}$ & $18.0 \pm 0.5 \mathrm{~d}$ & $158.3 \pm 2.5 c$ & $23.1 \pm 0.4 c$ & $73.9 \pm 1.6 c$ \\
\hline $100 \%$ ETc $\times$ Db-H & $21.4 \pm 0.5 c$ & $156.8 \pm 2.2 c$ & $22.8 \pm 0.3 c$ & $74.2 \pm 1.4 \mathrm{c}$ \\
\hline $60 \%$ ETc $\times$ Cn & $22.1 \pm 0.4 c$ & $212.6 \pm 2.9 b$ & $31.3 \pm 0.5 b$ & $97.6 \pm 1.9 a$ \\
\hline $60 \% \mathrm{ETc} \times \mathrm{GA}_{3}$ & $24.6 \pm 0.4 b$ & $259.7 \pm 3.4 \mathrm{a}$ & $41.8 \pm 0.6 \mathrm{a}$ & $83.2 \pm 1.8 b$ \\
\hline $60 \%$ ETc $\times$ Db-H & $27.8 \pm 0.6 a$ & $264.3 \pm 3.2 \mathrm{a}$ & $42.3 \pm 0.6 a$ & $81.9 \pm 1.8 b$ \\
\hline
\end{tabular}

** and * indicate respectively differences at $p \leq 0.05$ and $p \leq 0.01$ probability level. Means followed by the same letter in each column are not significantly different according to the LSD test $(p \leq 0.05)$.

\subsection{Antioxidant Defense System Components}

The contents of non-enzymatic antioxidants (glutathione (GSH), ascorbic acid (AsA), and $\alpha$-tocopherol ( $\alpha$.TOC)) (Table 5), and enzymatic antioxidant activities (superoxide dismutase (SOD), glutathione reductase (GR), catalase (CAT), and ascorbate peroxidase (APX)) (Table 6) were increased by $49 \%, 74 \%, 40 \%, 25 \%, 55 \%, 51 \%, 60 \%$, and $69 \%$ (seasons average), respectively, under the irrigation level of $60 \%$ of ETc compared to well-watered plants. However, foliar-applied $\mathrm{GA}_{3}$ or $\mathrm{Db}-\mathrm{H}$ substantially elevated the antioxidant capacity, while the total phenolic compounds were decreased. Under full irrigation, exogenously-applied $\mathrm{GA}_{3}$ or Db-H increased the activities of AsA (by $27 \%$ and $53 \%$ ), GSH (by $29 \%$ and $58 \%$ ), a.TOC (by $20 \%$ and $37 \%$ ), SOD (by $16 \%$ and $15 \%$ ), CAT (by $28 \%$ and $27 \%$ ), GR (by $26 \%$ and $25 \%$ ), and APX (by 14\% and 14\%) (seasons average), respectively, compared with the respective control, but not reached the activities obtained under drought stress.

Under water deficit stress, treatment with $\mathrm{GA}_{3}$ or $\mathrm{Db}-\mathrm{H}$ increased these antioxidant activities by $16 \%$ and $16 \%, 21 \%$ and $24 \%, 11 \%$ and $12 \%, 25 \%$ and $26 \%, 15 \%$ and $15 \%, 21 \%$ and $20 \%$, and $18 \%$ and $18 \%$ (seasons average), respectively, in relation to the corresponding control, and markedly exceeded those obtained under full irrigation (100\% of ETc) treatment. 
Table 5. Foliar application of some growth regulators (e.g., gibberellic acid; $\mathrm{GA}_{3}$ and diluted bee honey; $\mathrm{Db}-\mathrm{H}$ ) promoted non-enzymatic antioxidant contents of Vicia faba plants grown under well watering (100\% of crop evapotranspiration; ETc) or deficit irrigation $(60 \%$ of ETc).

\begin{tabular}{|c|c|c|c|c|}
\hline \multirow[t]{2}{*}{ Source of Variation } & $\begin{array}{c}\text { Ascorbate } \\
(\mu \mathrm{M} \text { per } \mathrm{g} \text { FW) }\end{array}$ & $\begin{array}{l}\text { Glutathione } \\
(\mu \mathrm{M} \text { per } \mathrm{g} F W)\end{array}$ & $\begin{array}{c}\alpha \text {-Tocopherol } \\
(\mu \mathrm{M} \text { per } \mathrm{g} \mathrm{DW})\end{array}$ & $\begin{array}{l}\text { Total Phenolic Compounds } \\
\text { (mg GAE per g DW) }\end{array}$ \\
\hline & \multicolumn{4}{|c|}{ Season of $2018 / 2019$} \\
\hline Irrigation (Ir) & $*$ & $* *$ & $*$ & $*$ \\
\hline $100 \%$ of ETc & $1.59 \pm 0.03 b$ & $0.88 \pm 0.02 b$ & $2.22 \pm 0.04 b$ & $8.10 \pm 0.27 \mathrm{~b}$ \\
\hline $60 \%$ of ETc & $2.28 \pm 0.04 a$ & $1.49 \pm 0.03 a$ & $3.10 \pm 0.05 a$ & $10.08 \pm 0.32 \mathrm{a}$ \\
\hline Regulators (Re) & * & $*$ & $*$ & * \\
\hline Control (Cn) & $1.69 \pm 0.03 c$ & $0.98 \pm 0.02 c$ & $2.39 \pm 0.04 c$ & $10.27 \pm 0.35 a$ \\
\hline $\mathrm{GA}_{3}$ & $1.99 \pm 0.04 b$ & $1.22 \pm 0.03 b$ & $2.72 \pm 0.04 b$ & $8.86 \pm 0.28 b$ \\
\hline $\mathrm{Db}-\mathrm{H}$ & $2.14 \pm 0.04 a$ & $1.36 \pm 0.03 a$ & $2.88 \pm 0.05 a$ & $8.15 \pm 0.26 c$ \\
\hline $\operatorname{Ir} \times \operatorname{Re}$ & * & * & * & * \\
\hline $100 \% \mathrm{ETc} \times \mathrm{Cn}$ & $1.23 \pm 0.02 \mathrm{e}$ & $0.64 \pm 0.01 \mathrm{e}$ & $1.89 \pm 0.03 \mathrm{e}$ & $8.12 \pm 0.30 c$ \\
\hline $100 \% \mathrm{ETc} \times \mathrm{GA}_{3}$ & $1.64 \pm 0.03 \mathrm{~d}$ & $0.89 \pm 0.02 \mathrm{~d}$ & $2.24 \pm 0.04 \mathrm{~d}$ & $8.10 \pm 0.26 c$ \\
\hline $100 \% \mathrm{ETc} \times \mathrm{Db}-\mathrm{H}$ & $1.91 \pm 0.03 c$ & $1.11 \pm 0.02 c$ & $2.53 \pm 0.05 c$ & $8.09 \pm 0.24 c$ \\
\hline $60 \% \mathrm{ETc} \times \mathrm{Cn}$ & $2.14 \pm 0.04 b$ & $1.32 \pm 0.03 b$ & $2.88 \pm 0.05 b$ & $12.42 \pm 0.39 a$ \\
\hline $60 \% \mathrm{ETc} \times \mathrm{GA}_{3}$ & $2.33 \pm 0.04 a$ & $1.55 \pm 0.04 a$ & $3.19 \pm 0.04 a$ & $9.62 \pm 0.30 b$ \\
\hline \multirow[t]{2}{*}{$60 \%$ ETc $\times$ Db-H } & $2.36 \pm 0.04 a$ & $1.60 \pm 0.03 a$ & $3.23 \pm 0.05 a$ & $8.21 \pm 0.27 c$ \\
\hline & \multicolumn{4}{|c|}{ Season of $2019 / 2020$} \\
\hline Irrigation (Ir) & $* *$ & $* *$ & $*$ & $*$ \\
\hline $100 \%$ of ETc & $1.47 \pm 0.02 b$ & $0.80 \pm 0.01 b$ & $2.39 \pm 0.05 b$ & $7.88 \pm 0.20 b$ \\
\hline $60 \%$ of ETc & $2.26 \pm 0.05 a$ & $1.43 \pm 0.03 a$ & $3.37 \pm 0.07 a$ & $9.85 \pm 0.25 a$ \\
\hline Regulators (Re) & * & * & * & $*$ \\
\hline Control (Cn) & $1.58 \pm 0.03 c$ & $0.95 \pm 0.02 c$ & $2.56 \pm 0.05 c$ & $10.05 \pm 0.27 \mathrm{a}$ \\
\hline $\mathrm{GA}_{3}$ & $1.93 \pm 0.04 b$ & $1.16 \pm 0.02 b$ & $2.94 \pm 0.06 b$ & $8.68 \pm 0.23 b$ \\
\hline $\mathrm{Db}-\mathrm{H}$ & $2.09 \pm 0.05 a$ & $1.25 \pm 0.02 \mathrm{a}$ & $3.15 \pm 0.07 a$ & $7.88 \pm 0.18 c$ \\
\hline $\operatorname{Ir} \times \operatorname{Re}$ & * & $*$ & * & * \\
\hline $100 \% \mathrm{ETc} \times \mathrm{Cn}$ & $1.19 \pm 0.01 \mathrm{e}$ & $0.66 \pm 0.01 \mathrm{e}$ & $1.98 \pm 0.04 \mathrm{e}$ & $7.89 \pm 0.21 c$ \\
\hline $100 \% \mathrm{ETc} \times \mathrm{GA}_{3}$ & $1.44 \pm 0.02 \mathrm{~d}$ & $0.79 \pm 0.01 \mathrm{~d}$ & $2.41 \pm 0.04 \mathrm{~d}$ & $7.91 \pm 0.19 c$ \\
\hline $100 \%$ ETc $\times$ Db-H & $1.79 \pm 0.04 c$ & $0.94 \pm 0.01 c$ & $2.79 \pm 0.06 c$ & $7.85 \pm 0.20 c$ \\
\hline $60 \%$ ETc $\times$ Cn & $1.97 \pm 0.04 b$ & $1.23 \pm 0.02 b$ & $3.14 \pm 0.06 b$ & $12.20 \pm 0.32 a$ \\
\hline $60 \% \mathrm{ETc} \times \mathrm{GA}_{3}$ & $2.41 \pm 0.05 a$ & $1.52 \pm 0.03 a$ & $3.47 \pm 0.07 a$ & $9.44 \pm 0.26 b$ \\
\hline $60 \%$ ETc $\times$ Db-H & $2.39 \pm 0.06 a$ & $1.55 \pm 0.03 a$ & $3.51 \pm 0.08 \mathrm{a}$ & $7.91 \pm 0.16 c$ \\
\hline
\end{tabular}

** and * indicate respectively differences at $p \leq 0.05$ and $p \leq 0.01$ probability level. Means followed by the same letter in each column are not significantly different according to the LSD test $(p \leq 0.05)$.

\subsection{Nutrient Contents}

In both seasons, faba bean plants exposed to a water deficit showed significant reductions in the contents of N (by 21\%), P (by 23\%), K (by 19\%), Fe (by 20\%), Mn (by 20\%) and $\mathrm{Zn}$ (by 20\%) in comparison to fully irrigated plants (Table 7). Regardless of irrigation levels, applying growth regulators $\left(\mathrm{GA}_{3}\right.$ or $\left.\mathrm{Db}-\mathrm{H}\right)$, especially $\mathrm{Db}-\mathrm{H}$, markedly increased the nutrient contents compared to untreated plants. Foliar-applied $\mathrm{GA}_{3}$ or $\mathrm{Db}-\mathrm{H}$ attenuated the adverse impact of drought on plant nutritional status. Where, $60 \%$ of $\mathrm{ETc} \times \mathrm{GA}_{3}$ or $\mathrm{Db}-\mathrm{H}$ treatment exhibited higher nutrient contents compared with $60 \%$ of ETc, recording values similar to or higher than values of full irrigated plants. The greatest nutrient contents were obtained under $100 \% \mathrm{ETc} \times \mathrm{Db}-\mathrm{H}$ treatment.

\subsection{Phytohormone Concentrations}

The phytohormone analyses (IAA, $\mathrm{GA}_{3}, \mathrm{CKs}$, and $\mathrm{ABA}$ ) displayed differences between the two irrigation regimes (Table 8). Drought-stressed plants exhibited lower IAA (by 23\%), $\mathrm{GA}_{3}$ (by 26\%), and CKs (by 25\%), and higher ABA (by 50\%) (seasons average) than nonstressed plants. As for the application of plant growth regulators, $\mathrm{GA}_{3}-$ or $(\mathrm{Db}-\mathrm{H})$-treated Vicia faba plants showed higher IAA, $\mathrm{GA}_{3}$, and $\mathrm{CKs}$ contents, and lower ABA content than untreated plants. 
Table 6. Foliar application of some growth regulators (e.g., gibberellic acid; $\mathrm{GA}_{3}$ and diluted bee honey; Db-H) promoted antioxidant enzyme activities of Vicia faba plants grown under well watering (100\% of crop evapotranspiration; ETc) or deficit irrigation (60\% of ETc).

\begin{tabular}{|c|c|c|c|c|}
\hline Source of Variation & $\begin{array}{c}\text { Superoxide } \\
\text { Dismutase }\left(\mathrm{A}_{564} \text { per }\right. \\
\text { min per } \mathrm{g} \text { Protein) }\end{array}$ & $\begin{array}{c}\text { Catalase }\left(A_{290} \text { per min }\right. \\
\text { per } g \text { Protein })\end{array}$ & $\begin{array}{c}\text { Glutathione } \\
\text { Reductase }\left(\mathrm{A}_{340} \text { per }\right. \\
\text { min per g Protein) }\end{array}$ & $\begin{array}{l}\text { Ascorbate Peroxidase } \\
\text { (A290 per min } \\
\text { per g Protein) }\end{array}$ \\
\hline & \multicolumn{4}{|c|}{ Season of $2018 / 2019$} \\
\hline Irrigation (Ir) & $* *$ & $* *$ & $* *$ & * \\
\hline $100 \%$ of ETc & $15.5 \pm 0.2 b$ & $56.5 \pm 0.7 \mathrm{~b}$ & $23.4 \pm 0.3 b$ & $68.4 \pm 0.8 b$ \\
\hline $60 \%$ of ETc & $23.4 \pm 0.4 \mathrm{a}$ & $85.5 \pm 0.8 \mathrm{a}$ & $37.1 \pm 0.4 \mathrm{a}$ & $93.2 \pm 0.7 \mathrm{a}$ \\
\hline Regulators (Re) & $*$ & $*$ & $*$ & $*$ \\
\hline Control (Cn) & $16.7 \pm 0.3 b$ & $63.4 \pm 0.7 \mathrm{~b}$ & $26.6 \pm 0.3 b$ & $71.6 \pm 0.7 \mathrm{~b}$ \\
\hline $\mathrm{GA}_{3}$ & $20.9 \pm 0.3 a$ & $75.3 \pm 0.8 \mathrm{a}$ & $32.2 \pm 0.4 \mathrm{a}$ & $85.4 \pm 0.8 \mathrm{a}$ \\
\hline $\mathrm{Db}-\mathrm{H}$ & $20.8 \pm 0.3 a$ & $74.3 \pm 0.8 \mathrm{a}$ & $32.1 \pm 0.4 \mathrm{a}$ & $85.4 \pm 0.7 \mathrm{a}$ \\
\hline $\operatorname{Ir} \times \operatorname{Re}$ & * & * & $*$ & $*$ \\
\hline $100 \% \mathrm{ETc} \times \mathrm{Cn}$ & $14.1 \pm 0.2 \mathrm{~d}$ & $48.5 \pm 0.6 \mathrm{~d}$ & $19.8 \pm 0.2 \mathrm{~d}$ & $62.1 \pm 0.8 \mathrm{~d}$ \\
\hline $100 \% \mathrm{ETc} \times \mathrm{GA}_{3}$ & $16.4 \pm 0.2 \mathrm{c}$ & $61.2 \pm 0.8 c$ & $25.1 \pm 0.3 c$ & $71.6 \pm 0.8 c$ \\
\hline $100 \% \mathrm{ETc} \times \mathrm{Db}-\mathrm{H}$ & $16.0 \pm 0.2 c$ & $59.8 \pm 0.7 c$ & $25.4 \pm 0.3 c$ & $71.4 \pm 0.7 \mathrm{c}$ \\
\hline $60 \% \mathrm{ETc} \times \mathrm{Cn}$ & $19.2 \pm 0.4 b$ & $78.3 \pm 0.8 b$ & $33.3 \pm 0.3 b$ & $81.1 \pm 0.6 b$ \\
\hline $60 \% \mathrm{ETc} \times \mathrm{GA}_{3}$ & $25.4 \pm 0.3 a$ & $89.4 \pm 0.8 \mathrm{a}$ & $39.2 \pm 0.5 a$ & $99.2 \pm 0.8 \mathrm{a}$ \\
\hline \multirow[t]{2}{*}{$60 \% \mathrm{ETc} \times \mathrm{Db}-\mathrm{H}$} & $25.6 \pm 0.4 a$ & $88.7 \pm 0.9 a$ & $38.8 \pm 0.5 a$ & $99.3 \pm 0.7 \mathrm{a}$ \\
\hline & \multicolumn{4}{|c|}{ Season of $2019 / 2020$} \\
\hline Irrigation (Ir) & $* *$ & $* *$ & $* *$ & $*$ \\
\hline $100 \%$ of ETc & $17.3 \pm 0.3 b$ & $52.9 \pm 0.5 b$ & $24.2 \pm 0.3 b$ & $64.5 \pm 0.7 b$ \\
\hline $60 \%$ of ETc & $27.4 \pm 0.4 a$ & $80.1 \pm 0.8 \mathrm{a}$ & $38.4 \pm 0.5 a$ & $85.4 \pm 1.0 \mathrm{a}$ \\
\hline Regulators (Re) & $*$ & $*$ & $*$ & $*$ \\
\hline Control (Cn) & $20.1 \pm 0.3 b$ & $58.3 \pm 0.7 \mathrm{~b}$ & $27.1 \pm 0.4 \mathrm{~b}$ & $69.2 \pm 0.8 b$ \\
\hline $\mathrm{GA}_{3}$ & $23.4 \pm 0.4 \mathrm{a}$ & $70.7 \pm 0.8 \mathrm{a}$ & $33.8 \pm 0.5 a$ & $77.9 \pm 0.9 a$ \\
\hline $\mathrm{Db}-\mathrm{H}$ & $23.7 \pm 0.4 \mathrm{a}$ & $70.7 \pm 0.6 \mathrm{a}$ & $33.1 \pm 0.4 \mathrm{a}$ & $78.0 \pm 0.8 \mathrm{a}$ \\
\hline $\operatorname{Ir} \times \operatorname{Re}$ & * & * & $*$ & $*$ \\
\hline $100 \% \mathrm{ETc} \times \mathrm{Cn}$ & $15.6 \pm 0.2 \mathrm{~d}$ & $44.2 \pm 0.5 \mathrm{~d}$ & $21.0 \pm 0.3 \mathrm{~d}$ & $59.7 \pm 0.7 d$ \\
\hline $100 \% \mathrm{ETc} \times \mathrm{GA}_{3}$ & $18.1 \pm 0.3 c$ & $57.1 \pm 0.6 \mathrm{c}$ & $26.2 \pm 0.3 c$ & $66.8 \pm 0.6 c$ \\
\hline $100 \% \mathrm{ETc} \times \mathrm{Db}-\mathrm{H}$ & $18.3 \pm 0.3 c$ & $57.4 \pm 0.5 c$ & $25.4 \pm 0.2 c$ & $67.1 \pm 0.7 c$ \\
\hline $60 \% \mathrm{ETc} \times \mathrm{Cn}$ & $24.5 \pm 0.3 b$ & $72.3 \pm 0.8 b$ & $33.1 \pm 0.4 b$ & $78.6 \pm 0.9 b$ \\
\hline $60 \% \mathrm{ETc} \times \mathrm{GA}_{3}$ & $28.7 \pm 0.4 \mathrm{a}$ & $84.2 \pm 0.9 \mathrm{a}$ & $41.3 \pm 0.6 \mathrm{a}$ & $88.9 \pm 1.1 \mathrm{a}$ \\
\hline $60 \% \mathrm{ETc} \times \mathrm{Db}-\mathrm{H}$ & $29.0 \pm 0.5 a$ & $83.9 \pm 0.7 a$ & $40.7 \pm 0.5 a$ & $88.8 \pm 0.9 a$ \\
\hline
\end{tabular}

** and * indicate respectively differences at $p \leq 0.05$ and $p \leq 0.01$ probability level. Means followed by the same letter in each column are not significantly different according to the LSD test $(p \leq 0.05)$.

The combination of these two factors (irrigation regimes and growth regulators) significantly increased IAA, $\mathrm{GA}_{3}$, and $\mathrm{CK}_{\mathrm{S}}$ contents, while decreased ABA content (Table 8). Interactive application of $\mathrm{GA}_{3}$ or $\mathrm{Db}-\mathrm{H}+$ full irrigation (100\% of ETc) increased IAA (by $20 \%$ and $55 \%$ ), $\mathrm{GA}_{3}$ (by $118 \% \%$ and $39 \%$ ), and CKs (by $36 \%$ and $68 \%$ ) (seasons average) compared to the respective control. Similarly, foliar-applied $\mathrm{GA}_{3}$ or $\mathrm{Db}-\mathrm{H}$ to plants subjected to water deficit (60\% of ETc) notably increased IAA (by $45 \%$ and $74 \%$ ), GA 3 (by $172 \%$ and $59 \%$ ), and CKs (by 48\% and 98\%), while decreased ABA (by 49\% and 59\%) (seasons average) compared to the corresponding control. 
Table 7. Foliar application of some growth regulators (e.g., gibberellic acid; $\mathrm{GA}_{3}$ and diluted bee honey; $\mathrm{Db}-\mathrm{H}$ ) promoted nutrient contents of Vicia faba plants grown under well watering (100\% of crop evapotranspiration; ETc) or deficit irrigation $(60 \%$ of ETc).

\begin{tabular}{|c|c|c|c|c|c|c|}
\hline $\begin{array}{l}\text { Source of } \\
\text { Variation }\end{array}$ & $\begin{array}{l}\text { Nitrogen (mg per } \\
\text { g Dry Weight) }\end{array}$ & $\begin{array}{l}\text { Phosphorus (mg } \\
\text { per g Dry } \\
\text { Weight) }\end{array}$ & $\begin{array}{l}\text { Potassium (mg } \\
\text { per g Dry } \\
\text { Weight) }\end{array}$ & $\begin{array}{l}\text { Iron (mg per g } \\
\text { Dry Weight) }\end{array}$ & $\begin{array}{c}\text { Manganese (mg } \\
\text { per g Dry } \\
\text { Weight) }\end{array}$ & $\begin{array}{c}\text { Zinc (mg per g } \\
\text { Dry Weight) }\end{array}$ \\
\hline & \multicolumn{6}{|c|}{ Season of $2018 / 2019$} \\
\hline Irrigation (Ir) & $*$ & $*$ & $*$ & $*$ & $*$ & $*$ \\
\hline $100 \%$ of ETc & $19.3 \pm 1.2 \mathrm{a}$ & $2.51 \pm 0.14 \mathrm{a}$ & $19.0 \pm 1.3 \mathrm{a}$ & $0.77 \pm 0.03 \mathrm{a}$ & $0.50 \pm 0.01 \mathrm{a}$ & $0.33 \pm 0.01 \mathrm{a}$ \\
\hline $60 \%$ of ETc & $15.3 \pm 1.3 b$ & $1.95 \pm 0.10 b$ & $16.3 \pm 1.0 \mathrm{~b}$ & $0.61 \pm 0.01 b$ & $0.41 \pm 0.01 b$ & $0.26 \pm 0.01 b$ \\
\hline Regulators (Re) & $*$ & $*$ & $*$ & $*$ & $*$ & $*$ \\
\hline Control (Cn) & $14.5 \pm 1.1 \mathrm{c}$ & $1.81 \pm 0.10 \mathrm{c}$ & $14.6 \pm 1.0 \mathrm{c}$ & $0.59 \pm 0.02 c$ & $0.38 \pm 0.01 c$ & $0.24 \pm 0.00 c$ \\
\hline $\mathrm{GA}_{3}$ & $18.2 \pm 1.2 b$ & $2.37 \pm 0.11 b$ & $18.2 \pm 1.1 b$ & $0.71 \pm 0.02 b$ & $0.48 \pm 0.01 b$ & $0.31 \pm 0.01 b$ \\
\hline $\mathrm{Db}-\mathrm{H}$ & $19.4 \pm 1.5 \mathrm{a}$ & $2.51 \pm 0.15 a$ & $20.3 \pm 1.5 a$ & $0.77 \pm 0.03 a$ & $0.52 \pm 0.02 \mathrm{a}$ & $0.35 \pm 0.01 \mathrm{a}$ \\
\hline $\operatorname{Ir} \times \operatorname{Re}$ & $*$ & $*$ & $*$ & * & $*$ & $*$ \\
\hline $100 \% \mathrm{ETc} \times \mathrm{Cn}$ & $16.8 \pm 0.9 c$ & $2.10 \pm 0.12 c$ & $17.2 \pm 1.1 \mathrm{c}$ & $0.68 \pm 0.02 c$ & $0.44 \pm 0.01 c$ & $0.29 \pm 0.00 c$ \\
\hline $100 \% \mathrm{ETc} \times \mathrm{GA}_{3}$ & $19.7 \pm 1.2 b$ & $2.56 \pm 0.12 b$ & $18.9 \pm 1.2 b$ & $0.76 \pm 0.02 b$ & $0.49 \pm 0.01 b$ & $0.33 \pm 0.01 b$ \\
\hline $100 \%$ ETc $\times$ Db-H & $21.5 \pm 1.5 \mathrm{a}$ & $2.88 \pm 0.17 \mathrm{a}$ & $20.9 \pm 1.7 a$ & $0.88 \pm 0.04 \mathrm{a}$ & $0.57 \pm 0.02 \mathrm{a}$ & $0.38 \pm 0.01 \mathrm{a}$ \\
\hline $60 \% \mathrm{ETc} \times \mathrm{Cn}$ & $12.1 \pm 1.3 \mathrm{~d}$ & $1.52 \pm 0.07 \mathrm{~d}$ & $11.9 \pm 0.8 \mathrm{~d}$ & $0.50 \pm 0.01 \mathrm{~d}$ & $0.31 \pm 0.00 \mathrm{~d}$ & $0.18 \pm 0.00 \mathrm{~d}$ \\
\hline $60 \% \mathrm{ETc} \times \mathrm{GA}_{3}$ & $16.7 \pm 1.2 \mathrm{c}$ & $2.18 \pm 0.10 c$ & $17.4 \pm 0.9 c$ & $0.66 \pm 0.01 c$ & $0.46 \pm 0.01 c$ & $0.28 \pm 0.01 c$ \\
\hline \multirow[t]{2}{*}{$60 \% \mathrm{ETc} \times \mathrm{Db}-\mathrm{H}$} & $17.2 \pm 1.4 \mathrm{c}$ & $2.14 \pm 0.12 \mathrm{c}$ & $19.6 \pm 1.2 b$ & $0.66 \pm 0.01 c$ & $0.47 \pm 0.01 c$ & $0.31 \pm 0.01 b$ \\
\hline & \multicolumn{6}{|c|}{ Season of $2019 / 2020$} \\
\hline Irrigation (Ir) & * & $*$ & * & $*$ & $*$ & $*$ \\
\hline $100 \%$ of ETc & $20.2 \pm 0.9 a$ & $2.41 \pm 0.11 \mathrm{a}$ & $20.9 \pm 1.0 \mathrm{a}$ & $0.80 \pm 0.02 a$ & $0.57 \pm 0.01 \mathrm{a}$ & $0.36 \pm 0.00 \mathrm{a}$ \\
\hline $60 \%$ of ETc & $15.9 \pm 0.6 b$ & $1.84 \pm 0.09 b$ & $15.9 \pm 0.8 b$ & $0.65 \pm 0.01 b$ & $0.44 \pm 0.00 \mathrm{~b}$ & $0.29 \pm 0.00 b$ \\
\hline Regulators (Re) & $*$ & $*$ & $*$ & $*$ & $*$ & $*$ \\
\hline Control (Cn) & $14.7 \pm 0.6 c$ & $1.70 \pm 0.07 c$ & $14.8 \pm 0.8 \mathrm{c}$ & $0.61 \pm 0.01 c$ & $0.41 \pm 0.00 c$ & $0.27 \pm 0.00 c$ \\
\hline $\mathrm{GA}_{3}$ & $18.9 \pm 0.7 \mathrm{~b}$ & $2.22 \pm 0.11 b$ & $18.9 \pm 1.0 \mathrm{~b}$ & $0.75 \pm 0.02 b$ & $0.53 \pm 0.01 b$ & $0.34 \pm 0.00 b$ \\
\hline $\mathrm{Db}-\mathrm{H}$ & $20.7 \pm 1.0 \mathrm{a}$ & $2.45 \pm 0.13 a$ & $21.6 \pm 1.0 \mathrm{a}$ & $0.83 \pm 0.02 \mathrm{a}$ & $0.58 \pm 0.01 \mathrm{a}$ & $0.37 \pm 0.01 \mathrm{a}$ \\
\hline $\operatorname{Ir} \times \operatorname{Re}$ & * & $*$ & * & $*$ & $*$ & $*$ \\
\hline $100 \% \mathrm{ETc} \times \mathrm{Cn}$ & $17.4 \pm 0.8 \mathrm{c}$ & $1.98 \pm 0.09 c$ & $16.9 \pm 0.9 c$ & $0.71 \pm 0.01 c$ & $0.50 \pm 0.00 c$ & $0.31 \pm 0.00 c$ \\
\hline $100 \% \mathrm{ETc} \times \mathrm{GA}_{3}$ & $19.9 \pm 0.8 b$ & $2.42 \pm 0.11 b$ & $20.4 \pm 0.9 b$ & $0.78 \pm 0.02 b$ & $0.56 \pm 0.01 b$ & $0.36 \pm 0.00 b$ \\
\hline $100 \%$ ETc $\times$ Db-H & $23.4 \pm 1.1 \mathrm{a}$ & $2.83 \pm 0.14 a$ & $25.3 \pm 1.2 \mathrm{a}$ & $0.91 \pm 0.02 a$ & $0.64 \pm 0.01 \mathrm{a}$ & $0.42 \pm 0.01 \mathrm{a}$ \\
\hline $60 \% \mathrm{ETc} \times \mathrm{Cn}$ & $11.9 \pm 0.4 \mathrm{~d}$ & $1.42 \pm 0.05 \mathrm{~d}$ & $12.6 \pm 0.6 \mathrm{~d}$ & $0.50 \pm 0.00 \mathrm{~d}$ & $0.32 \pm 0.00 \mathrm{~d}$ & $0.22 \pm 0.00 \mathrm{~d}$ \\
\hline $60 \% \mathrm{ETc} \times \mathrm{GA}_{3}$ & $17.8 \pm 0.6 c$ & $2.02 \pm 0.10 c$ & $17.4 \pm 1.0 \mathrm{c}$ & $0.72 \pm 0.01 c$ & $0.49 \pm 0.00 c$ & $0.32 \pm 0.00 c$ \\
\hline $60 \% \mathrm{ETc} \times \mathrm{Db}-\mathrm{H}$ & $17.9 \pm 0.9 c$ & $2.07 \pm 0.12 c$ & $17.8 \pm 0.8 \mathrm{c}$ & $0.74 \pm 0.01 b c$ & $0.51 \pm 0.01 c$ & $0.32 \pm 0.00 \mathrm{c}$ \\
\hline
\end{tabular}

${ }^{* *}$ and ${ }^{*}$ indicate respectively differences at $p \leq 0.05$ and $p \leq 0.01$ probability level. Means followed by the same letter in each column are not significantly different according to the LSD test $(p \leq 0.05)$.

Table 8. Foliar application of some growth regulators (e.g., gibberellic acid; $\mathrm{GA}_{3}$ and diluted bee honey; $\mathrm{Db}-\mathrm{H}$ ) promoted plant hormonal contents of Vicia faba plants grown under well watering (100\% of crop evapotranspiration; ETc) or deficit irrigation (60\% of ETc).

\begin{tabular}{|c|c|c|c|c|}
\hline \multirow[t]{2}{*}{ Source of Variation } & $\begin{array}{l}\text { Indole-3-Acetic Acid } \\
(\mu \mathrm{g} \text { per } \mathrm{g} \text { FW) }\end{array}$ & $\begin{array}{l}\text { Gibberellic Acid } \\
\text { ( } \mu \text { g per } \mathrm{g} \text { FW) }\end{array}$ & Cytokinins ( $\mu$ g per $\mathrm{g}$ FW) & $\begin{array}{l}\text { Abscisic Acid } \\
(\mu \mathrm{g} \text { per } \mathrm{g} \text { FW) }\end{array}$ \\
\hline & \multicolumn{4}{|c|}{ Season of $2018 / 2019$} \\
\hline Irrigation (Ir) & $*$ & $*$ & $*$ & $*$ \\
\hline $100 \%$ of ETc & $18.1 \pm 0.15 \mathrm{a}$ & $33.1 \pm 0.29 a$ & $24.6 \pm 0.18 \mathrm{a}$ & $4.23 \pm 0.05 b$ \\
\hline $60 \%$ of ETc & $14.2 \pm 0.14 b$ & $25.1 \pm 0.26 b$ & $18.4 \pm 0.16 b$ & $6.29 \pm 0.06 a$ \\
\hline Regulators (Re) & $*$ & $* *$ & $*$ & $*$ \\
\hline Control (Cn) & $12.2 \pm 0.10 \mathrm{c}$ & $18.5 \pm 0.20 c$ & $15.5 \pm 0.13 c$ & $7.43 \pm 0.07 a$ \\
\hline $\mathrm{GA}_{3}$ & $16.0 \pm 0.15 b$ & $41.9 \pm 0.40 \mathrm{a}$ & $22.0 \pm 0.18 b$ & $4.45 \pm 0.05 b$ \\
\hline $\mathrm{Db}-\mathrm{H}$ & $20.4 \pm 0.19 a$ & $26.9 \pm 0.23 b$ & $27.2 \pm 0.21 \mathrm{a}$ & $3.91 \pm 0.05 c$ \\
\hline $\operatorname{Ir} \times \operatorname{Re}$ & $*$ & $*$ & $*$ & $*$ \\
\hline $100 \% \mathrm{ETc} \times \mathrm{Cn}$ & $14.1 \pm 0.11 c$ & $22.4 \pm 0.19 \mathrm{~d}$ & $18.7 \pm 0.15 c$ & $5.22 \pm 0.06 b$ \\
\hline $100 \% \mathrm{ETc} \times \mathrm{GA}_{3}$ & $17.4 \pm 0.15 b$ & $45.6 \pm 0.39 a$ & $25.4 \pm 0.20 b$ & $3.77 \pm 0.04 \mathrm{e}$ \\
\hline $100 \%$ ETc $\times$ Db-H & $22.9 \pm 0.19 a$ & $31.2 \pm 0.28 c$ & $29.8 \pm 0.20 \mathrm{a}$ & $3.69 \pm 0.04 \mathrm{e}$ \\
\hline $60 \%$ ETc $\times$ Cn & $10.3 \pm 0.09 \mathrm{~d}$ & $14.6 \pm 0.20 \mathrm{e}$ & $12.2 \pm 0.11 \mathrm{~d}$ & $9.64 \pm 0.07 a$ \\
\hline $60 \% \mathrm{ETc} \times \mathrm{GA}_{3}$ & $14.5 \pm 0.15 c$ & $38.2 \pm 0.41 b$ & $18.5 \pm 0.15 c$ & $5.12 \pm 0.06 c$ \\
\hline $60 \% \mathrm{ETc} \times \mathrm{Db}-\mathrm{H}$ & $17.9 \pm 0.18 b$ & $22.6 \pm 0.18 \mathrm{~d}$ & $24.6 \pm 0.22 b$ & $4.12 \pm 0.05 \mathrm{~d}$ \\
\hline
\end{tabular}


Table 8. Cont

\begin{tabular}{|c|c|c|c|c|}
\hline \multirow[t]{2}{*}{ Source of Variation } & $\begin{array}{l}\text { Indole-3-Acetic Acid } \\
(\mu \mathrm{g} \text { per } \mathrm{g} \text { FW) }\end{array}$ & $\begin{array}{l}\text { Gibberellic Acid } \\
(\mu \mathrm{g} \text { per } \mathrm{g} \text { FW) }\end{array}$ & Cytokinins ( $\mu$ g per $g$ FW) & $\begin{array}{l}\text { Abscisic Acid } \\
(\mu \mathrm{g} \text { per } \mathrm{g} \text { FW) }\end{array}$ \\
\hline & \multicolumn{4}{|c|}{ Season of $2019 / 2020$} \\
\hline Irrigation (Ir) & $*$ & $*$ & $*$ & $* *$ \\
\hline $100 \%$ of ETc & $20.4 \pm 0.18 \mathrm{a}$ & $33.9 \pm 0.29 a$ & $24.2 \pm 0.20 \mathrm{a}$ & $3.75 \pm 0.04 b$ \\
\hline $60 \%$ of ETc & $15.8 \pm 0.14 b$ & $24.7 \pm 0.22 b$ & $18.2 \pm 0.19 b$ & $6.29 \pm 0.07 a$ \\
\hline Regulators (Re) & $*$ & $* *$ & $*$ & $*$ \\
\hline Control (Cn) & $14.1 \pm 0.13 c$ & $17.6 \pm 0.20 c$ & $15.0 \pm 0.14 c$ & $7.47 \pm 0.08 a$ \\
\hline $\mathrm{GA}_{3}$ & $18.1 \pm 0.15 b$ & $44.4 \pm 0.37 a$ & $20.9 \pm 0.19 b$ & $4.19 \pm 0.05 b$ \\
\hline $\mathrm{Db}-\mathrm{H}$ & $22.3 \pm 0.20 \mathrm{a}$ & $26.0 \pm 0.21 b$ & $27.7 \pm 0.26 a$ & $3.40 \pm 0.04 c$ \\
\hline $\operatorname{Ir} \times \operatorname{Re}$ & $*$ & $*$ & $*$ & $*$ \\
\hline $100 \% \mathrm{ETc} \times \mathrm{Cn}$ & $16.8 \pm 0.18 c$ & $21.6 \pm 0.22 \mathrm{~d}$ & $17.6 \pm 0.12 c$ & $4.98 \pm 0.05 b$ \\
\hline $100 \% \mathrm{ETc} \times \mathrm{GA}_{3}$ & $19.7 \pm 0.17 b$ & $50.2 \pm 0.45 a$ & $23.8 \pm 0.25 b$ & $3.48 \pm 0.03 c$ \\
\hline $100 \%$ ETc $\times$ Db-H & $24.8 \pm 0.19 a$ & $29.8 \pm 0.21 c$ & $31.2 \pm 0.22 \mathrm{a}$ & $2.78 \pm 0.03 \mathrm{~d}$ \\
\hline $60 \% \mathrm{ETc} \times \mathrm{Cn}$ & $11.3 \pm 0.08 \mathrm{~d}$ & $13.6 \pm 0.18 \mathrm{e}$ & $12.4 \pm 0.15 \mathrm{~d}$ & $9.96 \pm 0.11 a$ \\
\hline $60 \% \mathrm{ETc} \times \mathrm{GA}_{3}$ & $16.4 \pm 0.12 c$ & $38.5 \pm 0.29 b$ & $18.0 \pm 0.13 c$ & $4.89 \pm 0.06 b$ \\
\hline $60 \%$ ETc $\times$ Db-H & $19.7 \pm 0.21 b$ & $22.1 \pm 0.20 \mathrm{~d}$ & $24.2 \pm 0.30 \mathrm{~b}$ & $4.01 \pm 0.04 \mathrm{c}$ \\
\hline
\end{tabular}

** and * indicate respectively differences at $p \leq 0.05$ and $p \leq 0.01$ probability level. Means followed by the same letter in each column are not significantly different according to the LSD test $(p \leq 0.05)$.

\section{Discussion}

In dry regions including Egypt, drought stress is the major constraint to most crop plants, seriously limiting plant growth and productivity and regulating metabolism through complex and various mechanisms linked to plant metabolic pathways $[4,12]$. Under constant water deficit, plants are unable to withstand such stress through the available endogenous antioxidant defense system as in the case of the Vicia faba plants used in the current research. Therefore, Vicia faba plants must be supported by exogenous plant growth regulators that may stimulate several physio-biochemical processes, increase plant performance, and enhance resilience against water deficit stress. As presented in Table 10, $\mathrm{Db}-\mathrm{H}$ analysis showed that this promising tool for sustainable cultivation is a plant growth biostimulator for drought-stressed bean plants. $\mathrm{Db}-\mathrm{H}$ is rich in osmoprotectants (i.e., proline, total amino acids, and soluble sugars), different sugars, and mineral nutrients (i.e., $\mathrm{K}, \mathrm{P}, \mathrm{Mg}, \mathrm{Ca}, \mathrm{S}, \mathrm{Fe}$, $\mathrm{Mn}, \mathrm{Zn}, \mathrm{Cu}, \mathrm{I}, \mathrm{Na}$, and Se). Additionally, it has high values of vitamins (vitamin $\mathrm{C}$ and $\mathrm{B}$-group vitamins). Moreover, $\mathrm{Db}-\mathrm{H}$ possesses a high value of $\mathrm{DPPH}$ radical-scavenging activity $(88.2 \%)$, which is widely used for screening the antioxidant activity to prevent lipid peroxidation $[17,38]$, which confers the antioxidant property of $\mathrm{Db}-\mathrm{H}$. Moreover, exogenously-applied $\mathrm{GA}_{3}$ has been reported to induce various metabolic reactions to ameliorate abiotic stress $[27,44]$. Therefore, as shown in the current study, both $\mathrm{GA}_{3}$ and $\mathrm{Db}-\mathrm{H}$ have crucial mechanisms in favor of drought-stressed Vicia faba plants to boost their tolerance to drought stress.

In this study, lowering the irrigation level from $100 \%$ to $60 \%$ ETc restricted faba bean performance (growth and productivity; Table 1), impaired efficiency of photosynthesis machinery (Table 2), and disrupted leaf tissue stability (RWC and MSI; Table 3). As a result, lipid oxidation (MDA) was increased as a result of the excessive generation of oxidative stress markers $\left(\mathrm{H}_{2} \mathrm{O}_{2}\right.$ and $\left.\mathrm{O}_{2}{ }^{\bullet-}\right)$ (Table 3), associated with increased osmoprotectant compounds (Table 4), and upregulation of non-enzymatic (Table 5) and enzymatic antioxidants (Table 6), which cope with oxidative damage under drought stress [20]. Adverse effects exacerbated by water deficit may be ascribed to osmotic stress with loss of cell turgor and/or ROS overproduction under drought stress [11,23,45]. Nonetheless, foliar-applied $\mathrm{GA}_{3}$ or $\mathrm{Db}-\mathrm{H}$ ameliorated the adverse impacts caused by drought stress on the growth of faba bean plants, thus enhancing green pods yields to be comparable to those of wellwatered plants that had not been treated with growth regulators, thus increasing WUE. Under irrigation with $100 \%$ of ETc, the improvement in growth and yield of bean plants was more evident by $\mathrm{Db}-\mathrm{H}$ foliar spray resulting in higher WUE. The recovery of growth and productivity of drought-stressed Vicia faba plants by application of $\mathrm{GA}_{3}$ or $\mathrm{Db}-\mathrm{H}$ revealed that these growth regulators may include mechanisms to mitigate the effects of 
drought-induced stress. This is likely attributed to the growth-related metabolites of $\mathrm{Db}-\mathrm{H}$ dissolved substances such as proline, soluble sugars, amino acids, antioxidants, vitamins, and mineral nutrients, which support plants to restore their growth and development under drought stress $[46,47]$. Furthermore, $\mathrm{GA}_{3}$ upregulates the expression of genes (xyloglucan endotransglycosylases, expansins, and cyclin-dependent protein kinases) involved in increased cell division and elongation [48]. Moreover, $\mathrm{GA}_{3}$ induces osmoregulation by maintaining the osmotic potential, promoting enzyme activity, improving membrane permeability to facilitate mineral nutrient uptake and photosynthesis transportation $[22,49,50]$, thus stimulating plant growth and biomass production (Table 1).

RWC is a physiological indicator of available water content in favor of tissue metabolism, while the degree of membrane integrity can be assessed as MSI and EL [51,52]. Both growth regulators ( $\mathrm{Db}-\mathrm{H}$ and $\left.\mathrm{GA}_{3}\right)$ mediated recovery of stressed leaf tissues by increasing cell turgor (RWC) and membrane integrity (MSI), while ion leakage (EL) was reduced (Table 3). The improvement in RWC of drought-stressed plant tissues and cells helped maintain cell turgor through the accumulation of osmolytes such as proline, soluble sugars, and glycine betaine (Table 4) due to $\mathrm{Db}-\mathrm{H}$ and $\mathrm{GA}_{3}$ application and/or changes in elasticity of the cell wall $[9,53]$. This allowed for continued metabolic activities as effective mechanisms for drought tolerance in stressed faba bean plants. RWC enhanced by exogenous application of $\mathrm{Db}-\mathrm{H}$ or $\mathrm{GA}_{3}$ was closely related to increased WUE in faba bean plants. (Table 1). In this study, the increased protective compounds such as osmoprotectants, enzymatic antioxidants, and low molecular-weight antioxidants (Tables 4-6) by foliar-supplemented $\mathrm{Db}-\mathrm{H}$ or $\mathrm{GA}_{3}$ protected plasma membranes from lipid peroxidation (in term of MDA) by decreasing $\mathrm{H}_{2} \mathrm{O}_{2}$ and $\mathrm{O}_{2}{ }^{\bullet-}$ contents (Table 3). These findings may be related to improved MSI, decreased EL and photo-oxidation, and enhanced membrane integrity against oxidative damage $[38,46]$, and thus improved faba bean plant growth and outputs under water deficit stress.

In the current study, leaf photosynthetic pigment contents (total chlorophylls and carotenoids), photochemical activity, SPAD chlorophyll index, and photosynthetic efficiency $\left(\mathrm{F}_{\mathrm{v}} / \mathrm{F}_{\mathrm{m}}\right.$ and PI) were reduced while the irrigation water was reduced to $60 \%$ ETc, indicating chlorophyll degradation in chloroplasts and photoinhibition of PSII of waterstressed faba bean plants due to the damaging influences of ROS [54,55]. However, leaf photosynthetic pigment contents, photochemical activity, SPAD chlorophyll index, and photosynthetic efficiency (Table 2) were markedly improved by foliar-applied $\mathrm{Db}-\mathrm{H}$ [38] or $\mathrm{GA}_{3}$ [22]. These results may be related to maintaining cell membrane integrity and increasing leaf $\mathrm{RWC}$ by $\mathrm{Db}-\mathrm{H}$ or $\mathrm{GA}_{3}$ supplementation. Both $\mathrm{Db}-\mathrm{H}$ and $\mathrm{GA}_{3}$ likely mitigated the negative effects of drought, and faba bean plants responded to drought stress by up-regulation of osmoprotectants (Table 4), non-enzymatic (Table 5) and enzymatic antioxidants (Table 6) for ROS-scavenging to minimize lipid peroxidation. In line with our findings, $\mathrm{GA}_{3}$ supplementation improved leaf chlorophyll content in wheat [27] and maintained the photosynthetic efficiency of PSII in laurel seedlings [56]. Additionally, $\mathrm{Db}-\mathrm{H}$ is rich in nutrients to maintain intercellular hemostasis of ions required for photosynthetic biosynthesis, thus improving the efficiency of the photosynthetic machinery of Vicia faba plants.

Nutrients deficiency in plants that is attributed to the osmotic impact of water deficit stress and/or soil water deficit disturbs nutrient availability, uptake, translocation, and metabolism [9], which lead to the reduction of macro-and micro-nutrients contents in drought-stressed faba bean (Table 7). Nevertheless, foliar-applied $\mathrm{GA}_{3}$ or $\mathrm{Db}-\mathrm{H}$ induced ion hemostasis and increased mineral nutrient contents of drought-stressed plants. This may be attributed to that exogenous application of $\mathrm{GA}_{3}$ or $\mathrm{Db}-\mathrm{H}$ increased root uptake surfaces resulting from increased root system volume (data not shown), and/or increased accumulation of osmoprotectants (Table 4) to balance the osmotic pressure in organelles, thus mainlining cell turgor and improving nutritional status and water uptake [57].

In this work, the plant defense machinery including synthesis of osmoprotectants (proline, soluble sugars, glycine betaine, and total soluble protein; Table 4), and both 
non-enzymatic antioxidants contents (AsA, GSH, and $\alpha$.TOC; Table 7), and enzymatic antioxidants activities (SOD, CAT, GR, and APX; Table 6) increased in growth regulators ( $\mathrm{GA}_{3}$ and $\mathrm{Db}-\mathrm{H}$ )-treated plants. This positive situation protected faba bean plants from the deleterious impacts of water deficit stress by osmotic adjustment and ROS-scavenging [15,38]. Increased osmoprotectants are likely to lead to the uptake or breakdown of $\mathrm{Db}-\mathrm{H}$ as biostimulants, given that it is rich in osmoprotectant compounds (Table 9). Furthermore, $\mathrm{GA}_{3}$ regulates different genes that can modulate the osmotic ability to maintain cell enlargement through the accumulation of osmotically active solutes such as soluble sugar, soluble protein, free proline, and glycine betaine $[28,58]$. Our results showed that drought stress in combination with either of the growth regulators $\left(\mathrm{GA}_{3}\right.$ or $\left.\mathrm{Db}-\mathrm{H}\right)$ markedly improved the antioxidant defense system to enable Vicia faba plants to withstand drought stress through protection from oxidative damage as evidenced by the decreased contents of $\mathrm{MDA}, \mathrm{H}_{2} \mathrm{O}_{2}$, and $\mathrm{O}_{2}^{\bullet-}$ (Table 3).

Table 9. Some initial physical and chemical soil properties.

\begin{tabular}{|c|c|c|c|c|c|c|c|c|c|c|c|c|c|}
\hline \multirow{2}{*}{$\begin{array}{l}\text { Layer } \\
\text { (cm) }\end{array}$} & \multicolumn{4}{|c|}{ Particle Size Distribution } & \multirow{2}{*}{$\begin{array}{c}\text { Bulk } \\
\text { Density } \\
\left(\mathrm{g} \mathrm{cm}^{-3}\right)\end{array}$} & \multirow{2}{*}{$\begin{array}{c}\mathrm{K}_{\text {sat }} \\
\mathrm{Cm} \mathrm{h}^{-1}\end{array}$} & \multirow{2}{*}{ FC (\%) } & \multirow{2}{*}{$\begin{array}{l}\text { WP } \\
(\%)\end{array}$} & \multirow{2}{*}{$\begin{array}{l}\mathrm{AW} \\
(\%)\end{array}$} & \multirow{2}{*}{$\mathrm{pH}$} & \multirow{2}{*}{$\begin{array}{c}\text { ECe } \\
\left(\mathrm{dS} . \mathrm{m}^{-1}\right)\end{array}$} & \multirow{2}{*}{$\begin{array}{l}\text { OM } \\
(\%)\end{array}$} & \multirow{2}{*}{$\begin{array}{c}\mathrm{CaCO}_{3} \\
(\%)\end{array}$} \\
\hline & Sand \% & Silt \% & Clay \% & TC & & & & & & & & & \\
\hline $0-30$ & 20 & 38 & 42 & $\mathrm{CL}$ & 1.40 & 1.2 & 34.3 & 19.7 & 14.6 & 7.76 & 2.85 & 1.50 & 4.3 \\
\hline $30-60$ & 17 & 37 & 46 & $\mathrm{CL}$ & 1.36 & 0.9 & 32.2 & 19.1 & 13.1 & 7.75 & 2.98 & 1.10 & 4.2 \\
\hline
\end{tabular}

$\mathrm{TC}=$ Texture class, $\mathrm{CL}=$ Clay loam, $\mathrm{FC}=$ Field capacity, $\mathrm{WP}=$ Wilting point, $\mathrm{AW}=$ Available water, $\mathrm{OM}=$ Organic matter, and $\mathrm{K}_{\text {sat }}=$ Hydraulic conductivity.

It has been well demonstrated that phytohormones play an important role in various physiological, biochemical, and molecular processes in plants to mitigate drought stress [59], which was significantly increased by exogenous application of $\mathrm{GA}_{3}$ or $\mathrm{Db}-\mathrm{H}$, while ABA content was reduced (Table 8). In this study, Db-H promoted the contents of IAA, CKs, and $\mathrm{GA}_{3}$ in faba bean plants subjected to drought stress (Table 8), which could be attributed to the increased mineral nutrients required for the formation of protoplasm and phytohormones [38]. According to Semida and Rady [34], presoaking bean seeds with some extracts resulted in higher contents of IAA and $\mathrm{GA}_{3}$, while decreased ABA. Different genes are expressed after GAs treatment highlighting that GAs upregulated genes related to IAA and other genes related to ABA are down-regulated by GAs [58], while CKs have antagonistic roles against ABA [34]. Further, GAs-induced degradation of DELLA proteins is modulated by different signals such as salinity and drought, and other hormones [60], revealing that GAs regulate and crosstalk with other phytohormones to ameliorate the deleterious effects of drought stress. Water deficit stress disrupts the hormonal balance in plants, and thus, hormonal hemostasis may be a means for $\mathrm{GA}_{3}$-induced drought stress tolerance [25].

Finally, the negative effects of environmental foes may exceed the natural endurance of stressed plants. In this case, the components of a stressed plant's defense system do not meet adequate defense requirements, and therefore external use of auxiliary substances such as nutrients and other beneficial strategies increases the efficiency of antioxidant defenses, and thus plants can perform efficiently under adverse conditions of environmental foes [61-65].

\section{Materials and Methods}

\subsection{Experimental Location and Soil Properties}

Using a private farm (Fayoum; 29.3452 N, 30.5686 E, Egypt), two experiments were conducted at the field level during two consecutive winter seasons (2019 and 2020). The soil, 0.90-1.0 m deep, with loamy sand texture, which is classified as Typic Torripsamments, siliceous, hypothermic [66]. The soil physical and chemical properties were performed applying methods described in Klute [67] and Page et al. [68], and results are shown in Table 10. The electrical conductivity of the tested soil was $8.23 \mathrm{dS} \mathrm{m}^{-1}$, being saline soil according to the classification of Dahnke and Whitney [69]. 
Table 10. Physico-chemical composition of raw clover honey (on a fresh weight basis).

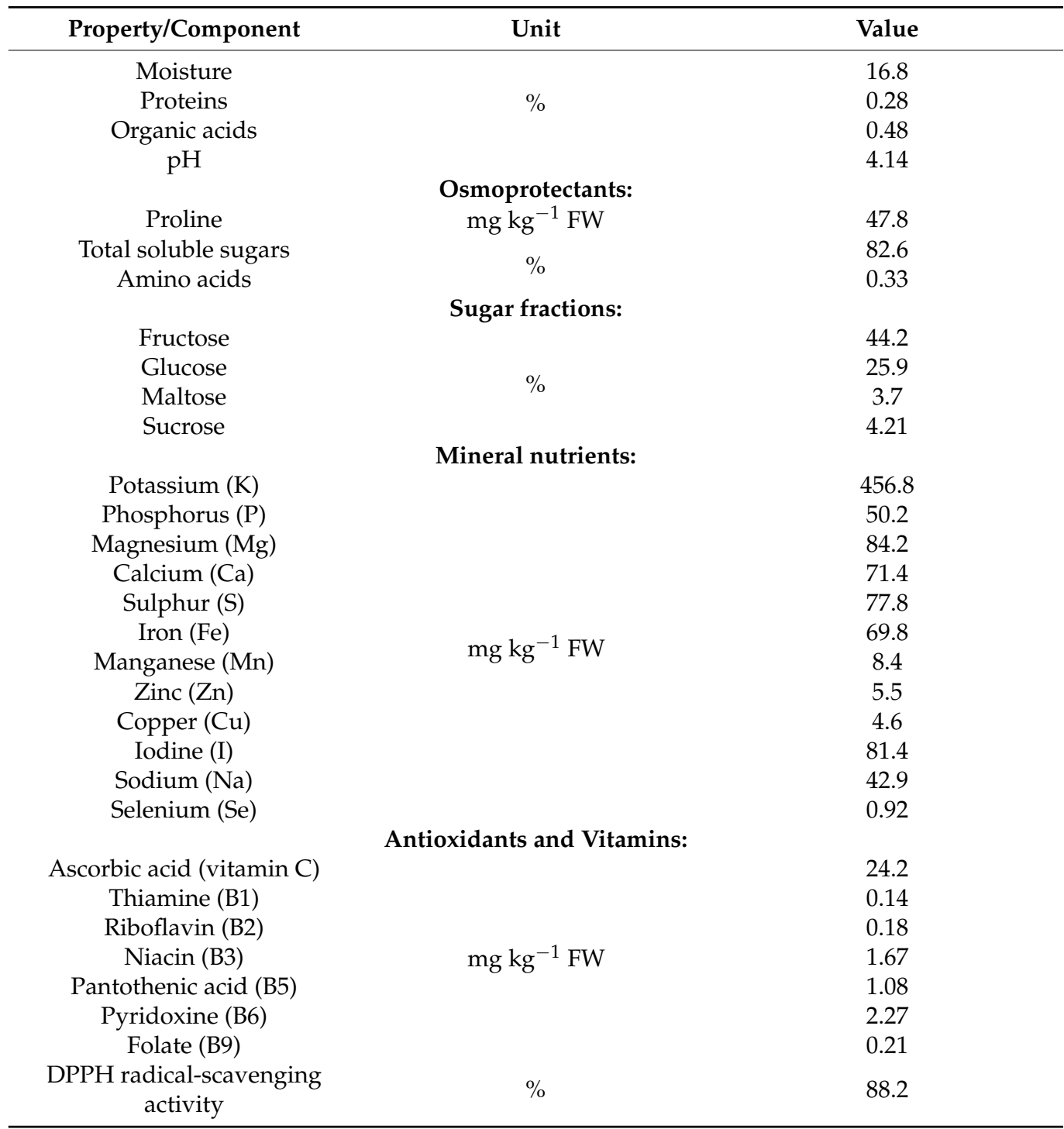

\subsection{Planting, Treatments, and Experimental Layout}

The seeds of Vicia faba (cv. Giza 40; widespread cultivar of faba bean in the study area based on the recommendation of the Egyptian Ministry of Agriculture) were secured from the Agricultural Research Center, Egypt. Firstly, the seeds were washed with distilled water then sterilized with sodium hypochlorite solution $(1 \%$; v/v) for roughly two min, once more the seed surface was cleaned from sterilization solution with distilled water after that were kept at room temperature to dry. The seeds were sown on October 20, for both seasons (2019 and 2020) in hills with plant and row spacing of $25 \times 70 \mathrm{~cm}$. Each plot area was $10.5 \mathrm{~m}^{2} ; 3.5 \mathrm{~m}$ length (5 rows) $\times 3 \mathrm{~m}$ width.

In this study, there are two treatment factors; including irrigation regimes and exogenous application of plant growth regulators. Two irrigation regimes were applied corresponding with $100 \%$ and $60 \%$ of the crop evapotranspiration (ETc). Gibberellic acid $\left(\mathrm{GA}_{3}\right)$ and diluted bee honey $(\mathrm{Db}-\mathrm{H})$ were applied at $20 \mathrm{mg}$ and $20 \mathrm{~g} \mathrm{~L}^{-1}$, respectively, as foliar spraying. These concentrations were selected based on our preliminary pot study (Table S1). The irrigation treatments were separated by a $1 \mathrm{~m}$ non-irrigated area. Until the full emergence of seedlings ( 15 days after planting; DAP), the faba bean plants were irrigated at $100 \%$ of ETc to ensure good plant establishment, thereafter the two irrigation treatments were initiated. These two irrigation treatments were chosen based on our preliminary pot study (Table S1). Fifteen days after the initiation of the irrigation treatments, 
$\mathrm{GA}_{3}$ and $\mathrm{Db}-\mathrm{H}$ were applied as foliar spraying in the early morning. Fifteen days after the first spraying, the second foliar spray was implemented for faba bean plants. Sprays were conducted to run-off, with the use of Tween-20 $(0.1 \%, \mathrm{v} / \mathrm{v})$ as a surfactant to ensure optimum penetration into leaf tissues. The plants $(n=200)$ in each experimental unit $\left(10.5 \mathrm{~m}^{2}\right)$ were sprayed with $2 \mathrm{~L}$ of spray solution, which was increased to $2.4 \mathrm{~L}$ for the second time of spraying. The experimental layout for each treatment was designed as a Randomized Split Plot with three replications. Different fertilizers (5 tons organic manure, $50 \mathrm{~kg}$ potassium humate, $75 \mathrm{~kg}$ of $\mathrm{P}_{2} \mathrm{O}_{5}$ using $\mathrm{Ca}\left(\mathrm{H}_{2} \mathrm{PO}_{4}\right)_{2} ; 15.5 \% \mathrm{P}_{2} \mathrm{O}_{5}, 60 \mathrm{~kg}$ of $\mathrm{K}_{2} \mathrm{O}$ using $\mathrm{K}_{2} \mathrm{SO}_{4} ; 48 \% \mathrm{~K}_{2} \mathrm{O}$, and $45 \mathrm{~kg}$ of N using $\left(\mathrm{NH}_{4}\right)_{2} \mathrm{SO}_{4} ; 21 \% \mathrm{~N}$ were added per hectare) and agronomic practices were applied following the recommendations of the Agricultural Research Center, Giza, Egypt.

\subsection{Irrigation Water Applied (IWA)}

The reference evapotranspiration (ETo) was given using the class A pan data $\left(\mathrm{E}_{\mathrm{pan}}\right.$; $\mathrm{mm}$ day $^{-1}$ ), adjacent to the experimental plots adjusted with appropriate pan coefficient $\left(K_{\text {pan }}\right)$ and the crop coefficient $\left(K_{c}\right)$ [70]. The ETc $\left(\mathrm{mm} \mathrm{day}^{-1}\right)$ was determined as the following formula [70]:

$$
\mathrm{ETc}=\mathrm{E}_{\mathrm{pan}} \times \mathrm{K}_{\mathrm{pan}} \times \mathrm{K}_{\mathrm{c}}
$$

Irrigation water applied (IWA) was computed with an equation as follows:

$$
\mathrm{IWA}=(\mathrm{A} \times \mathrm{ETc} \times \mathrm{Ii} \times \mathrm{Kr}) /[\mathrm{Ea} \times 1000 \times(1-\mathrm{LR})]
$$

where, IWA = irrigation water applied $\left(\mathrm{m}^{3}\right), \mathrm{A}=$ area of plots $\left(\mathrm{m}^{2}\right), \mathrm{ETc}=$ crop water requirements $(\mathrm{mm}$ per day), Ii = intervals of irrigation (day), $\mathrm{Kr}=$ covering factor, $\mathrm{Ea}=$ efficiency of application (\%), and $\mathrm{LR}=$ requirements for leaching.

The total irrigation water applied during both winter seasons was 3690 and $2214 \mathrm{~m}^{3}$ $\mathrm{ha}^{-1}$ in the 2019 season and 3754 and $2252 \mathrm{~m}^{3} \mathrm{ha}^{-1}$ in the 2020 season for 100 and $60 \%$ of ETc, respectively. The digital moisture meter sensors (HH2 type, Cambridge, CB5 0 EJ, UK) were utilized to record the water content of the tested soil every two days at different depths, $0-15$ and $15-30 \mathrm{~cm}$.

\subsection{Bee Honey Analysis for Physico-Chemical Composition}

Clover honey used in the current study was analysed for effective components and results are shown in Table 10. Moisture (\%), proline, and $\mathrm{pH}$ were assessed according to AOAC [71]. Quantities of sugars by High-Performance Liquid Chromatography (HPLC) were measured as the concentration of fructose, glucose, maltose and sucrose (\%) according to Bogdanov and Baumann [72]. Mineral nutrients were measured according to the methodology given in [73]. Ascorbic acid concentration was determined according to Mukherjee and Choudhuri [74]. Determination of the antioxidant activity was performed using 1,1-diphenyl-2-picrylhydrazyl (DPPH) assay as described by Lee et al. [75].

\subsection{Sampling and Measurements}

\subsubsection{Growth and Yield Characteristics, and WUE}

Plant growth characteristics were analyzed at sixty DAP in each season, 5 plants were selected, randomly, from each plot (main and sub-main plots). The number of branches and leaves for each plant was counted. The leaf area $\left(\mathrm{cm}^{2}\right)$ was measured using a held-hand planimeter (Planix 7, Tamaya Technics Inc. Tokyo, Japan). The shoot was weighed for each plant to determine to shoot fresh weight $(\mathrm{g})$. For recording shoot dry weight $(\mathrm{g})$, shoots were oven-dried at $70 \pm 2{ }^{\circ} \mathrm{C}$ until a constant weight was reached.

On the same date $(60 \mathrm{DAP})$, green pods yield parameters were recorded in terms of the number of green pods per plant and green pods' weight (ton) per hectare. These parameters 
of green pods yield were measured using the two outer rows of each experimental plot. The WUE was calculated as presented by Jensen [76]:

$$
\text { WUE }=\left[\text { Green pods yield }\left(\mathrm{kg} \mathrm{m}^{-2}\right)\right] /\left[\text { irrigation water applied }\left(\mathrm{m}^{-3} \mathrm{~m}^{-2}\right)\right]
$$

\subsubsection{Efficiency of the Photosynthetic Machinery}

Leaf photosynthetic pigment contents were determined in terms of chlorophylls and carotenoids based on the Arnon [77] procedures. Homogenization in $80 \%$ acetone (v/v) and centrifugation at $10,000 \times g$ for $10 \mathrm{~min}$ were implemented. The acetone extract solution absorption was recorded at 663,645 , and $470 \mathrm{~nm}$ in a UV-Visible spectrophotometer (UV-160A, Shimadzu, Japan).

Photochemical activity in fresh ear leaf was determined using the Ferricyanide technique as depicted by Jagendorf [78] with some modifications given in the Avron [79] method.

Using the SPAD-502 chlorophyll meter (Minolta, Osaka, Japan), the relative chlorophyll content (soil-plant analysis development (SPAD index) values) was measured. The measurements of chlorophyll "a" fluorescence were performed using a handy PEA chlorophyll fluorometer (Hansatech Instruments Ltd., Kings Lynn, UK). The maximum quantum yield of PSII $\left(\mathrm{F}_{\mathrm{v}} / \mathrm{F}_{\mathrm{m}}\right)$ was determined using the equation: $\mathrm{F}_{\mathrm{v}} / \mathrm{F}_{\mathrm{m}}=\left(\mathrm{F}_{\mathrm{m}}-\mathrm{F}_{0}\right) / \mathrm{F}_{\mathrm{m}}$ [80]. The photosynthesis performance index (PI) that quantifies multi-parameters as electron flow rate, absorption, trapping, and dissipation of excitation energy, was computed as described by Clark et al. [81].

\subsubsection{Leaf Tissue Stability and Oxidative Stress Biomarkers}

Using the fully enlarged upper leaves, the Osman and Rady [82] procedure was practiced to assess the leaf relative water content (RWC). Midribs were excluded and the leaf blades were divided into $2 \mathrm{~cm}$-diameter discs, which were immediately weighed (fresh mass). The discs were then saturated by deionized water for $24 \mathrm{~h}$ in the dark, gently surface-dried from the adhering water drops to record the turgid mass. To record dry mass, discs drying was implemented for $48 \mathrm{~h}$ under $70^{\circ} \mathrm{C}$, and the following equation was utilized for calculating RWC percentage:

$$
\operatorname{RWC}(\%)=[(\text { fresh mass }- \text { dry mass }) /(\text { turgid mass }- \text { dry mass })] \times 100
$$

Using the fully enlarged upper leaves, midribs were excluded and the leaf blades were divided into $0.2 \mathrm{~g}$ leaf pieces to evaluate leaf membrane stability index (MSI) [83]. A sample $(0.2 \mathrm{~g})$ was immersed in $10 \mathrm{ml}$ of ion-free water and $40{ }^{\circ} \mathrm{C}$ for $30 \mathrm{~min}$ was practiced to record $\mathrm{EC}_{1}$. Another $0.2 \mathrm{~g}$ sample was boiled for $10 \mathrm{~min}$ to record $\mathrm{EC}_{2}$. The following equation was utilized for calculating MSI percentage:

$$
\text { MSI }(\%)=[1-(E C 1 / E C 2)] \times 100
$$

Using fully enlarged upper leaves, midribs were excluded and the leaf blades were divided into discs to assess ions leaked from leaf tissue [83]. Using 20 discs immersed in $10 \mathrm{ml}$ of ion-free water, $\mathrm{EC}_{0}$ was recorded. $\mathrm{EC}_{1}$ was then measured after heating the tube content at $45-55^{\circ} \mathrm{C}$ for $30 \mathrm{~min}$. Then, the content of the tube was boiled for $10 \mathrm{~min}$ to record $\mathrm{EC}_{2}$. The following equation was utilized for calculating electrolyte leakage (EL) percentage:

$$
\mathrm{EL}(\%)=[(\mathrm{EC} 2-\mathrm{EC} 1) / \mathrm{EC} 3] \times 100
$$

Determination of lipid peroxidation that assessed as malondialdehyde (MDA), and the two biomarkers of oxidative stress; superoxide $\left(\mathrm{O}_{2}{ }^{\bullet-}\right)$, and hydrogen peroxide $\left(\mathrm{H}_{2} \mathrm{O}_{2}\right)$ contents were implemented applying the procedures of Madhava Rao and Sresty [84], Velikova et al. [85], and Kubiś [86], respectively. The contents of MDA were assessed applying an extinction coefficient $\left(155 \mathrm{mM}^{-1} \mathrm{~cm}^{-1}\right)$ and presented as $\mu \mathrm{mol} \mathrm{g}^{-1} \mathrm{FW}$. The $\mathrm{H}_{2} \mathrm{O}_{2}$ content ( $\mu \mathrm{mol} \mathrm{g}{ }^{-1} \mathrm{FW}$ ) was evaluated colorimetrically at $390 \mathrm{~nm}$ and the calculations were performed based on a proper standard curve. The $\mathrm{O}_{2}{ }^{\bullet-}$ content $\left(\mu \mathrm{mol} \mathrm{g}{ }^{-1} \mathrm{FW}\right)$ 
was evaluated using sample fragments $(1 \times 1 \mathrm{~mm}, 0.1 \mathrm{~g})$ that flooded using a buffer (Kphosphate, $10 \mathrm{mM}$, pH 7.8), which was mixed with each of NBT $(0.05 \%)$ and $\mathrm{NaN}_{3}(10 \mathrm{mM})$ for $60 \mathrm{~min}$ under $25^{\circ} \mathrm{C}$. The mixture was subjected to $85^{\circ} \mathrm{C}$ for $15 \mathrm{~min}$. The mixture was then cooled rapidly. The absorbance readings were taken at $580 \mathrm{~nm}$.

\subsubsection{Contents of Osmoprotectant Compounds}

Using toluene, extraction of proline was practiced and at $520 \mathrm{~nm}$, the absorbance was recorded [87]. Leaf content ( $\mu \mathrm{g}$ proline $\mathrm{g}^{-1} \mathrm{FW}$ ) of proline was calculated using a suitable standard curve. Glycine betaine (GB) content was estimated under acidic conditions through monitoring formed periodide crystals colorimetrically (at $365 \mathrm{~nm}$ ) after reaction of the mixture with a reagent (cold $\mathrm{KI}-\mathrm{I}_{2}$ ) [88]. By utilizing a professional method [89], extraction (with $96 \%$ ethyl alcohol), and determination of the content of total soluble sugars $\left(\mathrm{mg} \mathrm{g}^{-1} \mathrm{DW}\right)$. The reaction of the ethanolic extract $(100 \mu \mathrm{L})$ was implemented with $150 \mathrm{mg}$ of anthrone as a reagent prepared, freshly, in $100 \mathrm{~mL} \mathrm{H}_{2} \mathrm{SO}_{4}, 72 \%$. Then, the mixture was boiled for $10 \mathrm{~min}$ and readings were taken at $625 \mathrm{~nm}$ after cooling. The procedures described in Bradford [90] were used to determine total soluble protein content.

\subsubsection{Contents of Non-Enzymatic Antioxidant Compounds}

Ascorbate (AsA) was determined in the tissue of the upper fully-expanded leaf after the homogenization in $\mathrm{HPO}_{3}$ (ice-cold, 5\%) contained $1 \mathrm{mM}$ EDTA. The produced homogenates were centrifuged at 4,000 $\times \mathrm{g}$ for $20 \mathrm{~min}$, and supernatants were used to estimate AsA [91]. Determination of glutathione (GSH) was performed [92] with a minor modification [93] and a known concentration of GSH was used as a standard curve. $\alpha-$ Tocopherol ( $\alpha$-TOC) was detected according to the method of Ching and Mohamed [94] and Konings et al. [95]. The total leaf content of phenolic compounds was assessed by the Folin-Ciocalteu method [96] functioning gallic acid as a standard. At $725 \mathrm{~nm}$, the absorbance readings were recorded and the total phenolic contents were presented as $\mathrm{mg}$ gallic acid equivalents (GAE) $\mathrm{g}^{-1}$ dry weight, computed from a standard curve prepared with gallic acid.

\subsubsection{Activities of Antioxidant Enzymes}

The fully enlarged upper leaves were used to extract enzymes in $0.5 \mathrm{~g}$. An ice-cold buffer, pH 7.0 (e.g., 100 mM K-phosphate, which contained 1\% PVP) was utilized with a prechilled (cleaned) mortar and pestle to macerate leaf samples. The obtained homogenates were transferred for the centrifugation process at $12,000 \times g$ for $0.25 \mathrm{~h}$ under $4{ }^{\circ} \mathrm{C}$. The obtained supernatants were the enzymatic extracts, which were utilized for assaying the activities of catalase (CAT), glutathione reductase (GR), and ascorbate peroxidase (APX). Using the method detailed in Aebi [97], assaying of the CAT activity (Unit mg ${ }^{-1}$ protein) was performed using a spectrophotometer apparatus at $240 \mathrm{~nm}$. To assay the ability of the enzyme to decompose the $\mathrm{H}_{2} \mathrm{O}_{2}$ for $2 \mathrm{~min}, 2 \mathrm{~mL}$ of the reaction mixture of a P-buffer (50 mM, pH 6.0), EDTA $(0.1 \mathrm{mM}), \mathrm{H}_{2} \mathrm{O}_{2}(0.02 \mathrm{M})$, and $0.1 \mathrm{~mL}$ of the enzymatic extract was applied, and an extinction coefficient $\left(39.4 \mathrm{mM}^{-1} \mathrm{~cm}^{-1}\right)$ was also applied. The Nakano and Asada [98] method was applied to assay the APX activity (Unit $\mathrm{mg}^{-1}$ protein). Using spectrophotometer, $2 \mathrm{~mL}$ mixture (P-buffer $(50 \mathrm{mM}, \mathrm{pH} 7.5)$, EDTA $(100 \mu \mathrm{M})$, AsA $(300 \mu \mathrm{M})$, $0.1 \mathrm{~mL} \mathrm{H}_{2} \mathrm{O}_{2}$, and $0.1 \mathrm{~mL}$ enzyme extract) was observed for $2 \mathrm{~min}$ at $290 \mathrm{~nm}$, and $2.8 \mathrm{mM}^{-1}$ $\mathrm{cm}^{-1}$ was applied as an extinction coefficient. The Foster and Hess [99] method was applied to assess the GR activity (Unit $\mathrm{mg}^{-1}$ protein) by monitoring (for $3 \mathrm{~min}$ at $340 \mathrm{~nm}$ ) the changes that occurred in the reading of the reaction mixture (K-phosphate buffer $(0.1 \mathrm{M}$, $\mathrm{pH} 7.0)$, EDTA $(100 \mu \mathrm{M}), \mathrm{NADPH}(0.5 \mathrm{mM})$, GSSG $(0.1 \mathrm{mM})$, and $0.1 \mathrm{~mL}$ enzyme extract).

Homogenization with ice was performed for frozen samples $(500 \mathrm{mg})$ and the homogenization solution was $10 \mathrm{~mL}$ of $50 \mathrm{mM} \mathrm{L}^{-1}$ HEPES buffer and $0.1 \mathrm{mM} \mathrm{L}^{-1} \mathrm{Na}_{2}$ EDTA ( $\mathrm{pH}$ 7.6). To obtain a crude extract, centrifugation was practiced for homogenates for a quarter of an hour at $15,000 \times g$ under $4{ }^{\circ} \mathrm{C}$, which was functioned for assaying protein and superoxide dismutase (SOD). Overnight, dialyzing of crude extract was performed 
against a diluted homogenizing solution to eradicate the interference in SOD assay from substances having low molecular weights. The protein-dye binding method [90] was functioned to assess the concentration of soluble protein against a standard (bovine serum albumin). Assaying the SOD (EC 1.15.1.1) activity was implemented through inhibiting NBT photochemical reduction under practicing the Yu and Rengel [100] method.

\subsubsection{Contents of Nutrient Elements}

Digestion process was performed for the dried leaf samples with a mixture consisting of perchloric and nitric acids (at 1: 3, $\mathrm{v} / \mathrm{v}$, respectively). Using the previous digestion solution, assessments of $\mathrm{N}, \mathrm{P}$, and $\mathrm{K}^{+}$contents were performed. Determination of $\mathrm{N}$ was performed using the micro-Kjeldahl apparatus (Ningbo Medical Instruments Co., Ningbo, China) following [101]. The P content was assessed following the blue color method [102] whereby molybdenum was used to reduce molybdophosphoric in sulfuric acid while reducing to exclude arsenic. The $\mathrm{K}^{+}$content was assessed utilizing a flame photometer (Perkin-Elmer Model 52-A, Glenbrook, Stamford, CT, USA) device as depicted in the methods of Page et al. [68]. Micronutrients ( $\mathrm{Zn}, \mathrm{Mn}$, and Fe) contents were detected in dried leaf samples according to Johnson and Ulrich [103] with atomic absorption spectroscopy under checking against standard reference samples (NIST, USA).

\subsubsection{Contents of Plant Hormones}

The phytohormones; indole-3-acetic acid (IAA), gibberellic acid $\left(\mathrm{GA}_{3}\right)$, cytokinins (CKs) profiling were implemented based on the procedures of gas chromatographymass spectrometry (GC-MS) methods improved by Nehela et al. [104] with minor adjustments [26]. Fresh leaves $(0.1 \mathrm{~g})$ sample was extracted in ice-cold extraction solvent $(2 \mathrm{~mL}$; methanol/water/ $\mathrm{HCl}(6 \mathrm{~N}) ; 80 / 19.9 / 0.1 ; v / v / v)$. Then, the extract was centrifuged at $25,000 \times \mathrm{g}, 4^{\circ} \mathrm{C}$ for $5 \mathrm{~min}$. Supernatants were collected and concentrated to $50 \mu \mathrm{L}$ under $\mathrm{N}$ stream and then stored at $-80^{\circ} \mathrm{C}$ until analysis. For IAA, $50 \mu \mathrm{L}$ of the supernatant was derivatized with $40 \mu \mathrm{L}$ of MCF then concentrated to $20 \mu \mathrm{L}$ under $\mathrm{N}$ stream and $0.5 \mathrm{mg}$ of $\mathrm{Na}_{2} \mathrm{SO}_{4}$ were added to dry the organic phase. For CKs and $\mathrm{GA}_{3}, 50 \mu \mathrm{L}$ from the supernatant was dried and derivatized with $100 \mu \mathrm{L}$ of N-Methyl-N-(trimethylsilyl) trifluoroacetamide (MSTFA) by heating at $85^{\circ} \mathrm{C}$ for $45 \mathrm{~min}$. For GC-MS analysis, $1 \mu \mathrm{L}$ was injected into the GC-MS running in the selective ion mode (SIM-mode). All samples and phytohormone standards were analysed using a Clarus 680 GC with SQ8-T Mass Spectrometer system (Perkin Elmer, Waltham, MA, USA) fitted with an Elite-5MS capillary column (low bleed, $30 \mathrm{~m} \times 0.25 \mathrm{~mm} \times 0.025 \mu \mathrm{m}$ film thickness; Perkin Elmer, Waltham, MA, USA). Helium was the carrier gas with a flow rate of $1 \mathrm{~mL} \mathrm{~min}^{-1}$. The temperature program for IAA was as the following: the column was held at $50{ }^{\circ} \mathrm{C}$ for $3 \mathrm{~min}$, and then increased to $200{ }^{\circ} \mathrm{C}$ at a rate of $4{ }^{\circ} \mathrm{C} \mathrm{min}-1$, held for $5 \mathrm{~min}$. While, the program for CKs and $\mathrm{GA}_{3}$ was as the following: the column was held at $60^{\circ} \mathrm{C}$ for $2 \mathrm{~min}$ and then increased to $160^{\circ} \mathrm{C}$ at $20^{\circ} \mathrm{C}$ $\min ^{-1}$, and finally to $290^{\circ} \mathrm{C}$ at $5^{\circ} \mathrm{C} \mathrm{min}^{-1}$. The injector and the detector temperatures were set at $250^{\circ} \mathrm{C}$ and $260^{\circ} \mathrm{C}$, respectively. The TurboMass software version 6.1 (Perkin Elmer, Waltham, MA, USA) was used to analyze chromatograms. Identification of IAA, $\mathrm{CKs}$ and $\mathrm{GA}_{3}$ was performed by comparing their retention time, linear retention indices (LRIs) and the selected ions with those of authentic standards. Extraction and estimation of the content of abscisic acid (ABA) were implemented using high-performance liquid chromatography (HPLC) as outlined by Ünyayar et al. [105].

\subsection{Statistical Tests}

The data were analyzed based on the GLM procedures of the GENSTAT software (VSN International Ltd, Oxford, UK). All data were subjected to the combined analysis and the mean differences were compared with the least significant difference (LSD) test at $5 \%$ probability $(p \leq 0.05)$ level. The analyzed results are presented as the mean \pm standard error. 


\section{Conclusions}

The current study exhibits differences in physiological, biochemical, and metabolic responses among the $(\mathrm{Db}-\mathrm{H})$ - or $\mathrm{GA}_{3}$-treated and untreated faba bean plants. Exogenous application of $\mathrm{Db}-\mathrm{H}$ or $\mathrm{GA}_{3}$ markedly elevated the level of non-enzymatic and enzymatic antioxidants and osmoprotectants (proline, glycine betaine, soluble sugars, and soluble protein) as well as increased the phytohormones (indole-3-acetic acid and gibberellic acid and cytokinins), this associated with the reduction of malondialdehyde (MDA) and abscisic acid (ABA). Foliar applied Db-H or $\mathrm{GA}_{3}$ improved the nutrients status, tissue health, leaf photosynthetic pigments, and photosynthetic efficiency leading to higher growth and productivity (yield and water use efficiency) of drought-stressed faba bean plants. Therefore, the application of these growth regulators $\left(\mathrm{Db}-\mathrm{H}\right.$ and $\left.\mathrm{GA}_{3}\right)$ was identified to be an effective strategy to mitigate the damage effects of irrigation water deficits for sustainable faba bean production in arid and semi-arid areas.

Supplementary Materials: The following are available online at https: / www.mdpi.com/article/ 10.3390/plants10040748/s1, Table S1: A preliminary pot study conducted to identify the optimal concentration of diluted bee-honey $(\mathrm{Db}-\mathrm{H})$ and gibberellic acid $\left(\mathrm{GA}_{3}\right)$, as well as identifying the drought threshold of faba bean (Giza 40 cultivar) for the main study.

Author Contributions: Conceptualization, M.M.R., S.H.K.B., T.A.A.E.-M. and M.A.S.E.-Y.; Data curation, M.M.R., S.H.K.B. and T.A.A.E.-M.; Formal analysis, M.M.R., S.H.K.B., T.A.A.E.-M., M.A.S.E.Y. and A.A.; Investigation, M.M.R., S.H.K.B., T.A.A.E.-M., M.A.S.E.-Y., E.F.A., F.A.S.H. and A.A.; Methodology, M.M.R., S.H.K.B., T.A.A.E.-M. and A.A.; Resources, M.M.R., S.H.K.B., T.A.A.E.-M., M.A.S.E.-Y., E.F.A., F.A.S.H. and A.A.; Software, M.M.R., S.H.K.B., T.A.A.E.-M., M.A.S.E.-Y., E.F.A., F.A.S.H. and A.A.; Writing-original draft, M.M.R., S.H.K.B., T.A.A.E.-M., M.A.S.E.-Y., E.F.A., F.A.S.H. and A.A.; Writing-review and editing, M.M.R., E.F.A., F.A.S.H. and A.A. All authors have read and agreed to the published version of the manuscript.

Funding: The Deanship of Scientific Research at Taif University through the research number TURSP2020/143 is acknowledged.

Institutional Review Board Statement: Not applicable.

Informed Consent Statement: Not applicable.

Data Availability Statement: The data presented in this study are available upon request from the corresponding author.

Acknowledgments: The authors are thankful to the Taif University Researchers Supporting Project number (TURSP-2020/143), Taif University, Taif, Saudi Arabia for providing the financial support and research facilities.

Conflicts of Interest: The authors declare no conflict of interest.

Sample Availability: Samples of faba bean plant are available from the authors.

\section{References}

1. Semida, W.M.; Taha, R.S.; Abdelhamid, M.T.; Rady, M.M. Foliar-applied $\alpha$-tocopherol enhances salt-tolerance in Vicia faba L. plants grown under saline conditions. S. Afr. J. Bot. 2014, 95, 24-31. [CrossRef]

2. Karkanis, A.; Ntatsi, G.; Lepse, L.; Fernández, J.A.; Vågen, I.M.; Rewald, B.; Alsina, I.; Kronberga, A.; Balliu, A.; Olle, M.; et al.. Faba bean cultivation-Revealing novel managing practices for more sustainable and competitive European cropping systems. Front. Plant Sci. 2018, 9, 1-14. [CrossRef]

3. Khazaei, H.; Vandenberg, A. Seed Mineral Composition and Protein Content of Faba Beans (Vicia faba L.) with Contrasting Tannin Contents. Agronomy 2020, 10, 511. [CrossRef]

4. Semida, W.M.; Abdelkhalik, A.; Rady, M.O.A.; Marey, R.A.; Abd El-Mageed, T.A. Exogenously applied proline enhances growth and productivity of drought stressed onion by improving photosynthetic efficiency, water use efficiency and up-regulating osmoprotectants. Sci. Hortic. 2020, 272, 109580. [CrossRef]

5. Abdelkhalik, A.; Pascual, B.; Nájera, I.; Domene, M.A.; Baixauli, C.; Pascual-Seva, N. Effects of deficit irrigation on the yield and irrigation water use efficiency of drip-irrigated sweet pepper (Capsicum annuum L.) under Mediterranean conditions. Irrig. Sci. 2020, 38, 89-104. [CrossRef] 
6. AQUASTAT. AQUASTAT_FAO's Global Information System on Water and Agriculture; Food and Agriculture Organization: Rome, Italy, 2019.

7. Pal, S.; Zhao, J.; Khan, A.; Yadav, N.S.; Batushansky, A.; Barak, S.; Rewald, B.; Fait, A.; Lazarovitch, N.; Rachmilevitch, S. Paclobutrazol induces tolerance in tomato to deficit irrigation through diversified effects on plant morphology, physiology and metabolism. Sci. Rep. 2016, 6, 1-13. [CrossRef]

8. Abdelkhalik, A.; Pascual-Seva, N.; Nájera, I.; Domene, M.Á.; Baixauli, C.; Pascual, B. Effect of deficit irrigation on the productive response of drip-irrigated onion (Allium cepa L.) in mediterranean conditions. Hortic. J. 2019, 88, 488-498. [CrossRef]

9. Farooq, M.; Wahid, A.; Kobayashi, N.; Fujita, D.; Basra, S.M.A. Plant drought stress: Effects, mechanisms and management. Agron. Sustain. Dev. 2009, 29, 185-212. [CrossRef]

10. Fahad, S.; Bajwa, A.A.; Nazir, U.; Anjum, S.A.; Farooq, A.; Zohaib, A.; Sadia, S.; Nasim, W.; Adkins, S.; Saud, S.; et al.. Crop production under drought and heat stress: Plant responses and management options. Front. Plant Sci. 2017, 8, 1-16. [CrossRef] [PubMed]

11. Upadhyaya, H.; Sahoo, L.; Panda, S.K. Molecular Physiology of Osmotic Stress in Plants. In Molecular Stress Physiology of Plants; Rout, G.R., Das, A.B., Eds.; Springer: New Delhi, India, 2013; pp. 179-192. ISBN 978-81-322-0807-5.

12. Zhu, J. Abiotic Stress Signaling and Responses in Plants. Cell 2016, 167, 313-324. [CrossRef]

13. Abd El-Mageed, T.A.; Rady, M.M.; Taha, R.S.; Abd El Azeam, S.; Simpson, C.R.; Semida, W.M. Effects of integrated use of residual sulfur-enhanced biochar with effective microorganisms on soil properties, plant growth and short-term productivity of Capsicum annuum under salt stress. Sci. Hortic. 2020, 261, 108930. [CrossRef]

14. Rady, M.M.; Taha, S.S.; Kusvuran, S. Integrative application of cyanobacteria and antioxidants improves common bean performance under saline conditions. Sci. Hortic. 2018, 233, 61-69. [CrossRef]

15. Khalid, A.; Aftab, F. Effect of exogenous application of IAA and GA3 on growth, protein content, and antioxidant enzymes of Solanum tuberosum L. grown in vitro under salt stress. Vitr. Cell. Dev. Biol. Plant 2020, 56, 377-389. [CrossRef]

16. Abd El-Mageed, T.A.; Semida, W.M.; Mohamed, G.F.; Rady, M.M. Combined effect of foliar-applied salicylic acid and deficit irrigation on physiological-anatomical responses, and yield of squash plants under saline soil. S. Afr. J. Bot. 2016, 106, 8-16. [CrossRef]

17. Taha, R.S.; Alharby, H.F.; Bamagoos, A.A.; Medani, R.A.; Rady, M.M. Elevating tolerance of drought stress in Ocimum basilicum using pollen grains extract; a natural biostimulant by regulation of plant performance and antioxidant defense system. S. Afr. J. Bot. 2020, 128, 42-53. [CrossRef]

18. Rady, M.M.; Belal, H.E.E.; Gadallah, F.M.; Semida, W.M. Selenium application in two methods promotes drought tolerance in Solanum lycopersicum plant by inducing the antioxidant defense system. Sci. Hortic. 2020, 266, 109290. [CrossRef]

19. Sitohy, M.Z.; Desoky, E.S.M.; Osman, A.; Rady, M.M. Pumpkin seed protein hydrolysate treatment alleviates salt stress effects on Phaseolus vulgaris by elevating antioxidant capacity and recovering ion homeostasis. Sci. Hortic. 2020, 271, 109495. [CrossRef]

20. Hasanuzzaman, M.; Borhannuddin Bhuyan, M.H.M.; Anee, T.I.; Parvin, K.; Nahar, K.; Al Mahmud, J.; Fujita, M. Regulation of ascorbate-glutathione pathway in mitigating oxidative damage in plants under abiotic stress. Antioxidants 2019, 8, 384. [CrossRef]

21. Turner, N.C. Turgor maintenance by osmotic adjustment: 40 years of progress. J. Exp. Bot. 2018, 69, 3223-3233. [CrossRef]

22. Miceli, A.; Moncada, A.; Sabatino, L.; Vetrano, F. Effect of gibberellic acid on growth, yield, and quality of leaf lettuce and rocket grown in a floating system. Agronomy 2019, 9, 382. [CrossRef]

23. Taiz, L.; Zeiger, E. Plant Physiology, 3rd ed.; Sinauer Associates: Sunderland, MA, USA, 2002; ISBN 0878938230.

24. Ashraf, M.; Karim, F.; Rasul, E. Interactive effects of gibberellic acid (GA3) and salt stress on growth, ion accumulation and photosynthetic capacity of two spring wheat (Triticum aestivum L.) cultivars differing in salt tolerance. Plant Growth Regul. 2002, 36, 49-59. [CrossRef]

25. Iqbal, M.; Ashraf, M. Gibberellic acid mediated induction of salt tolerance in wheat plants: Growth, ionic partitioning, photosynthesis, yield and hormonal homeostasis. Environ. Exp. Bot. 2013, 86, 76-85. [CrossRef]

26. Rady, M.M.; Talaat, N.B.; Abdelhamid, M.T.; Shawky, B.T.; Desoky, E.S.M. Maize (Zea mays L.) grains extract mitigates the deleterious effects of salt stress on common bean (Phaseolus vulgaris L.) growth and physiology. J. Hortic. Sci. Biotechnol. 2019, 94, 777-789. [CrossRef]

27. Al Mahmud, J.; Biswas, P.K.; Nahar, K.; Fujita, M.; Hasanuzzaman, M. Exogenous application of gibberellic acid mitigates drought-induced damage in spring wheat. Acta Agrobot. 2019, 72. [CrossRef]

28. Li, Z.; Lu, G.Y.; Zhang, X.K.; Zou, C.S.; Cheng, Y.; Zheng, P.Y. Improving drought tolerance of germinating seeds by exogenous application of gibberellic acid (GA3) in rapeseed (Brassica napus L.). Seed Sci. Technol. 2010, 38, 432-440. [CrossRef]

29. Hasanuzzaman, M.; Fujita, M.; Oku, H.; Islam, M.T. Plant Tolerance to Environmental Stress: Role of Phytoprotectants; CRC Press: Boca Raton, FL, USA, 2019; Volume 4, ISBN 9781138559172.

30. Verma, V.; Ravindran, P.; Kumar, P.P. Plant hormone-mediated regulation of stress responses. BMC Plant Biol. 2016, 16, 1-10. [CrossRef] [PubMed]

31. Rouphael, Y.; Colla, G. Synergistic Biostimulatory Action: Designing the Next Generation of Plant Biostimulants for Sustainable Agriculture. Front. Plant Sci. 2018, 9, 1-7. [CrossRef]

32. Teklić, T.; Parađiković, N.; Špoljarević, M.; Zeljković, S.; Lončarić, Z.; Lisjak, M. Linking abiotic stress, plant metabolites, biostimulants and functional food. Ann. Appl. Biol. 2020, 1-23. [CrossRef] 
33. Lucini, L.; Rouphael, Y.; Cardarelli, M.; Canaguier, R.; Kumar, P.; Colla, G. The effect of a plant-derived biostimulant on metabolic profiling and crop performance of lettuce grown under saline conditions. Sci. Hortic. 2015, 182, 124-133. [CrossRef]

34. Semida, W.M.; Rady, M.M. Presoaking application of propolis and maize grain extracts alleviates salinity stress in common bean (Phaseolus vulgaris L.). Sci. Hortic. 2014, 168, 210-217. [CrossRef]

35. Abd El-Mageed, T.A.; Semida, W.M.; Rady, M.M. Moringa leaf extract as biostimulant improves water use efficiency, physiobiochemical attributes of squash plants under deficit irrigation. Agric. Water Manag. 2017, 193, 46-54. [CrossRef]

36. Desoky, E.S.M.; Elrys, A.S.; Rady, M.M. Integrative moringa and licorice extracts application improves Capsicum annuum fruit yield and declines its contaminant contents on a heavy metals-contaminated saline soil. Ecotoxicol. Environ. Saf. 2019, 169, 50-60. [CrossRef]

37. Di Mola, I.; Cozzolino, E.; Ottaiano, L.; Giordano, M.; Rouphael, Y.; Colla, G.; Mori, M. Effect of Vegetal- and Seaweed ExtractBased Biostimulants on Agronomical and Leaf Quality Traits of Plastic Tunnel-Grown Baby Lettuce under Four Regimes of Nitrogen Fertilization. Agronomy 2019, 9, 571. [CrossRef]

38. Semida, W.M.; Abd El-Mageed, T.A.; Hemida, K.; Rady, M.M. Natural bee-honey based biostimulants confer salt tolerance in onion via modulation of the antioxidant defence system. J. Hortic. Sci. Biotechnol. 2019, 94, 632-642. [CrossRef]

39. Van Oosten, M.J.; Pepe, O.; De Pascale, S.; Silletti, S.; Maggio, A. The role of biostimulants and bioeffectors as alleviators of abiotic stress in crop plants. Chem. Biol. Technol. Agric. 2017, 4, 1-12. [CrossRef]

40. Shin, H.; Ustunol, Z. Carbohydrate composition of honey from different floral sources and their influence on growth of selected intestinal bacteria: An in vitro comparison. Food Res. Int. 2005, 38, 721-728. [CrossRef]

41. Saxena, S.; Gautam, S.; Sharma, A. Physical, biochemical and antioxidant properties of some Indian honeys. Food Chem. 2010, 118, 391-397. [CrossRef]

42. Inés, M.; Craig, A.; Ordoñez, R.; Zampini, C.; Sayago, J.; Bedascarrasbure, E.; Alvarez, A.; Salomón, V.; Maldonado, L. LWT—Food Science and Technology Physico chemical and bioactive properties of honeys from Northwestern Argentina. LWT Food Sci. Technol. 2011, 44, 1922-1930. [CrossRef]

43. Bulgari, R.; Franzoni, G.; Ferrante, A. Biostimulants application in horticultural crops under abiotic stress conditions. Agronomy 2019, 9, 306. [CrossRef]

44. Wang, Z.; Liu, L.; Cheng, C.; Ren, Z.; Xu, S.; Li, X. GAI functions in the plant response to dehydration stress in arabidopsis Thaliana. Int. J. Mol. Sci. 2020, 21, 819. [CrossRef]

45. Osakabe, Y.; Osakabe, K.; Shinozaki, K.; Tran, L.-S.P. Response of plants to water stress. Front. Plant Sci. 2014, 5, 86. [CrossRef]

46. Alzahrani, Y.; Rady, M.M. Compared to antioxidants and polyamines, the role of maize grain-derived organic biostimulants in improving cadmium tolerance in wheat plants. Ecotoxicol. Environ. Saf. 2019, 182, 109378. [CrossRef]

47. Desoky, E.M.; El-maghraby, L.M.M.; Awad, A.E.; Abdo, A.I.; Rady, M.M.; Semida, W.M. Fennel and ammi seed extracts modulate antioxidant defence system and alleviate salinity stress in cowpea (Vigna unguiculata). Sci. Hortic. 2020, 272, 109576. [CrossRef]

48. Sun, T. Gibberellin signal transduction in stem elongation \& leaf growth. In Plant Hormones; Davies, P.J., Ed.; Springer: Dordrecht, The Netherlands, 2004; pp. 308-328. ISBN 9781402026867.

49. Azuma, T.; Ueno, S.; Uchida, N.; Yasuda, T. Gibberellin-induced elongation and osmoregulation in internodes of floating rice. Physiol. Plant. 1997, 99, 517-522. [CrossRef]

50. Wood, A.; Paleg, L.G. The Influence of Gibberellic Acid on the Permeability of Model Membrane Systems. Plant Physiol. 1972, 50, 103-108. [CrossRef] [PubMed]

51. Yan, W.; Zhong, Y.; Shangguan, Z. A meta-analysis of leaf gas exchange and water status responses to drought. Sci. Rep. 2016, 6, 1-9. [CrossRef] [PubMed]

52. Zhao, H.; Zhang, K.; Zhou, X.; Xi, L.; Wang, Y.; Xu, H.; Pan, T.; Zou, Z. Melatonin alleviates chilling stress in cucumber seedlings by up-regulation of CsZat12 and modulation of polyamine and abscisic acid metabolism. Sci. Rep. 2017, 7, 1-12. [CrossRef] [PubMed]

53. Slabbert, M.M.; Krüger, G.H.J. Antioxidant enzyme activity, proline accumulation, leaf area and cell membrane stability in water stressed Amaranthus leaves. S. Afr. J. Bot. 2014, 95, 123-128. [CrossRef]

54. Guidi, L.; Lo Piccolo, E.; Landi, M. Chlorophyll fluorescence, photoinhibition and abiotic stress: Does it make any difference the fact to be a C3 or C4 species? Front. Plant Sci. 2019, 10, 1-11. [CrossRef]

55. Muhammad, I.; Shalmani, A.; Ali, M.; Yang, Q.-H.; Ahmad, H.; Li, F.B. Mechanisms Regulating the Dynamics of Photosynthesis Under Abiotic Stresses. Front. Plant Sci. 2021, 11, 1-25. [CrossRef]

56. Aktas, L.Y.; Akca, H.; Altun, N.; Battal, P. Phytohormone levels of drought acclimated laurel seedlings in semiarid conditions. Gen. Appl. Plant Physiol. 2008, 34, 203-214.

57. Singh, M.; Kumar, J.; Singh, S.; Singh, V.P.; Prasad, S.M. Roles of osmoprotectants in improving salinity and drought tolerance in plants: A review. Rev. Environ. Sci. Biotechnol. 2015, 14, 407-426. [CrossRef]

58. Munteanu, V.; Gordeev, V.; Martea, R.; Duca, M. Effect of gibberellin cross talk with other phytohormones on cellular growth and mitosis to endoreduplication transition. Int. J. Adv. Res. Biol. Sci. 2014, 1, 1-18.

59. Ullah, A.; Manghwar, H.; Shaban, M.; Khan, A.H.; Akbar, A.; Ali, U.; Ali, E.; Fahad, S. Phytohormones enhanced drought tolerance in plants: A coping strategy. Environ. Sci. Pollut. Res. 2018, 25, 33103-33118. [CrossRef] [PubMed]

60. Kaya, C.; Tuna, A.L.; Alves, A.A.C. Gibberellic acid improves water deficit tolerance in maize plants. Aust. J. Crop Sci. 2006, 28, 331-337. [CrossRef] 
61. ElSayed, A.I.; Boulila, M.; Rafudeen, M.S.; Sengupta, S.; Rady, M.M. Melatonin regulatory mechanisms and phylogenetic analyses implying new sequences of melatonin biosynthesis related genes extracted from peanut under salinity stress. Plants $2020,9,854$. [CrossRef] [PubMed]

62. Seleiman, M.F.; Semida, W.M.; Rady, M.M.; Mohamed, G.F.; Hemida, K.A.; Alhammad, B.A.; Hassan, M.M.; Shami, A. Sequenced Antioxidants Application Rectifies Ion Imbalance and Strengthens Antioxidant Systems in Salt-stressed Cucumber. Plants 2020, 9, 1783. [CrossRef]

63. Taha, R.S.; Seleiman, M.F.; Alotaibi, M.; Alhammad, B.A.; Rady, M.M.; Mahdi, A.H.A. Exogenous potassium treatments elevate salt tolerance and performances of Glycine max by boosting antioxidant defense system under actual saline field conditions. Agronomy 2020, 10, 1741. [CrossRef]

64. Desoky, E.S.; Mansour, E.; Ali, M.M.A.; Yasin, M.A.T.; Abdul-Hamid, M.I.E.; Rady, M.M.; Ali, E.F. Exogenously used 24epibrassinolide promotes drought tolerance in maize hybrids by improving plant and water productivity in an arid environment. Plants 2021, 10, 354. [CrossRef] [PubMed]

65. Semida, W.M.; Abdelkhalik, A.; Mohamed, G.F.; Abd El-Mageed, T.A.; Abd El-Mageed, S.A.; Rady, M.M.; Ali, E.F. Foliar Application of Zinc Oxide Nanoparticles Promotes Drought Stress Tolerance in Eggplant (Solanum melongena L.). Plants 2021, 10, 421. [CrossRef] [PubMed]

66. Soil Survey Staff USDA. Keys to Soil Taxonomy, 12th ed.; USDA-NRCS: Washington, DC, USA, $2014 ;$ ISBN 0926487221.

67. Klute, A. Methods of Soil Analysis: Part. 1 Physical and Mineralogical Methods, 2nd ed.; The American Society of Agronomy, Inc.: Madison, WI, USA; Soil Science Society of America, Inc.: Madison, WI, USA, 1986.

68. Page, A.L.; Miller, R.H.; Keeney, D.R. Methods of Soil Analysis Part. 2. Chemical and Microbiological Properties; American Society of Agronomy, Inc.: Madison, WI, USA, 1982.

69. Dahnke, W.C.; Whitney, D.A. Measurement of soil salinity. In Recommended Chemical Soil Test Procedures for the North Central Region. North Central Regional Publication 221.; Dahnke, W.C., Ed.; North Dakota Agricultural Experiment Station Bulletin: Fargo, ND, USA, 1988; Volume 499, pp. 32-34.

70. Allen, R.G.; Pereira, L.S.; Raes, D.; Smith, M. Crop Evapotranspiration: Guidelines for Computing Crop Requirements; Irrigation and Drainage Paper No. 56; Food and Agriculture Organization of the United Nations: Rome, Italy, 1988.

71. AOAC. Official methods of analysis of AOAC international. In Association of Official Analysis Chemists International; Horwitz, W., Ed.; AOAC: Rockville, MD, 1995; Volume II, pp. 1058-1059.

72. Bogdanov, S.; Baumann, E. Bestimmung von Honigzuckern mit HPLC. Mitt. Geb. Lebensm. Hyg. 1988, 79, $198-206$.

73. Chapman, H.D.; Pratt, P.F. Methods of Analysis for Soil, Plants and Water; Division of Agricultural Science, University of California: Berkeley, CA, USA, 1961; pp. 60-61, 150-179.

74. Mukherjee, S.P.; Choudhuri, M.A. Implications of water stress-induced changes in the levels of endogenous ascorbic acid and hydrogen peroxide in Vigna seedlings. Physiol. Plant. 1983, 58, 166-170. [CrossRef]

75. Lee, S.; Kim, J.; Jeong, S.; Kim, D. Effect of far-infrared radiation on the antioxidant activity of rice hulls. J. Agric. Food Chem. 2003, 51, 4400-4403. [CrossRef] [PubMed]

76. Jensen, M.E. Design and Operation of Farm Irrigation Systems; American Society of Agricultural Engineers: St. Joseph, MI, USA, $1983 ;$ p. 827.

77. Arnon, D.I. Copper enzymes in isolated chloroplasts. Polyphenol oxidase in Beta vulgaris L. Plant Physiol. 1949, $24,1-16$. [CrossRef] [PubMed]

78. Jagendorf, A.T. Oxidation and reduction of pyridine nucleotides by purified chloroplasts. Arch. Biochem. Biophys. 1956, 62, 141-150. [CrossRef]

79. Avron, M. Photophosphorylation by Swis- chard chloroplasts chloroplasts. Biochim. Biophys. Acta 1960, 40, 257-272. [CrossRef]

80. Maxwell, K.; Johnson, G.N. Chlorophyll fluorescence-A practical guide. J. Exp. Bot. 2000, 51, 659-668. [CrossRef]

81. Clark, A.J.; Landolt, W.; Bucher, J.B.; Strasser, R.J. Beech (Fagus sylvatica) response to ozone exposure assessed with a chlorophyll a fluorescence performance index. Environ. Pollut. 2000, 109, 501-507. [CrossRef]

82. Osman, A.S.; Rady, M.M. Effect of humic acid as an additive to growing media to enhance the production of eggplant and tomato transplants. J. Hortic. Sci. Biotechnol. 2014, 89, 237-244. [CrossRef]

83. Rady, M.M. Effect of 24-epibrassinolide on growth, yield, antioxidant system and cadmium content of bean (Phaseolus vulgaris L.) plants under salinity and cadmium stress. Sci. Hortic. 2011, 129, 232-237. [CrossRef]

84. Madhava Rao, K.V.; Sresty, T.V.S. Antioxidative parameters in the seedlings of pigeonpea (Cajanus cajan (L.) Millspaugh) in response to Zn and Ni stresses. Plant Sci. 2000, 157, 113-128. [CrossRef]

85. Velikova, V.; Yordanov, I.; Edreva, A. Oxidative stress and some antioxidant systems in acid rain-treated bean plants protective role of exogenous polyamines. Plant Sci. 2000, 151, 59-66. [CrossRef]

86. Kubiś, J. Exogenous spermidine differentially alters activities of some scavenging system enzymes, $\mathrm{H}_{2} \mathrm{O}_{2}$ and superoxide radical levels in water-stressed cucumber leaves. J. Plant Physiol. 2008, 165, 397-406. [CrossRef]

87. Bates, L.S.; Waldren, R.P.; Teare, I.D. Rapid determination of free proline for water-stress studies. Plant Soil 1973, $207,205-207$. [CrossRef]

88. Grieve, C.M.; Grattan, S.R. Rapid assay for determination of water soluble quaternary ammonium compounds. Plant Soil 1983, 70, 303-307. [CrossRef] 
89. Irigoyen, J.J.; Einerich, D.W.; Sánchez-Díaz, M. Water stress induced changes in concentrations of proline and total soluble sugars in nodulated alfalfa (Medicago sativa) plants. Physiol. Plant. 1992, 84, 55-60. [CrossRef]

90. Bradford, M.M. A rapid and sensitive method for the quantitation of microgram quantities of protein utilizing the principle of protein-dye binding. Anal. Biochem. 1976, 72, 248-254. [CrossRef]

91. Huang, C.; He, W.; Guo, J.; Chang, X.; Su, P.; Zhang, L. Increased sensitivity to salt stress in an ascorbate-deficient Arabidopsis mutant. J. Exp. Bot. 2005, 56, 3041-3049. [CrossRef]

92. Yu, C.W.; Murphy, T.M.; Lin, C.H. Hydrogen peroxide-induced chilling tolerance in mung beans mediated through ABAindependent glutathione accumulation. Funct. Plant Biol. 2003, 30, 955-963. [CrossRef]

93. Paradiso, A.; Berardino, R.; De Pinto, M.C.; Sanità Di Toppi, L.; Storelli, M.M.; Tommasi, F.; De Gara, L. Increase in ascorbateglutathione metabolism as local and precocious systemic responses induced by cadmium in durum wheat plants. Plant Cell Physiol. 2008, 49, 362-374. [CrossRef] [PubMed]

94. Ching, L.S.; Mohamed, S. Alpha-tocopherol content in 62 edible tropical plants. J. Agric. Food Chem. 2001, 49, 3101-3105. [CrossRef] [PubMed]

95. Konings, E.J.M.; Roomans, H.H.S.; Beljaars, P.R. Liquid Chromatographic Determination of Tocopherols and Tocotrienols in Margarine, Infant Foods, and Vegetables. J. AOAC Int. 1996, 79, 902-906. [CrossRef] [PubMed]

96. Makkar, H.P.S.; Becker, K.; Abel, H.; Pawelzik, E. Nutrient contents, rumen protein degradability and antinutritional factors in some colour- and white-flowering cultivars of Vicia faba beans. J. Sci. Food Agric. 1997, 75, 511-520. [CrossRef]

97. Aebi, H. Catalase in Vitro. Methods Enzymol. 1984, 105, 121-126. [CrossRef]

98. Nakano, Y.; Asada, K. Hydrogen peroxide is scavenged by ascorbate-specific peroxidase in spinach chloroplasts. Plant Cell Physiol. 1981, 22, 867-880. [CrossRef]

99. Foster, J.G.; Hess, J.L. Responses of Superoxide Dismutase and Glutathione Reductase Activities in Cotton Leaf Tissue Exposed to an Atmosphere Enriched in Oxygen. Plant Physiol. 1980, 66, 482-487. [CrossRef]

100. Yu, Q.; Rengel, Z. Drought and salinity differentially influence activities of superoxide dismutases in narrow-leafed lupins. Plant Sci. 1999, 142, 1-11. [CrossRef]

101. AOAC. Official Methods of Analysis of the Association of Official Agricultural Chemists, 6th ed.; AOAC: Washington, DC, USA, 1995.

102. Jackson, M.L. Soil Chemical Analysis; Prentice Hall of India Pvt. Ltd.: New Delhi, India, 1967.

103. Johnson, C.M.; Ulrich, A. Analytical Methods for Use in Plant Analysis; Bulletin (Agricultural Experiment Station, Berkeley, Calif.); University of California: Berkeley, CA, USA, 1959.

104. Nehela, Y.; Hijaz, F.; Elzaawely, A.A.; El-Zahaby, H.M.; Killiny, N. Phytohormone profiling of the sweet orange (Citrus sinensis (L.) Osbeck) leaves and roots using GC-MS-based method. J. Plant Physiol. 2016, 199, 12-17. [CrossRef] [PubMed]

105. Ünyayar, S.; Topcuoglu, S.F.; Ünyayar, A. A modified method for extraction and identification of indole-3-acetic acid (IAA), gibberellic acid (GA3), abscisic acid (ABA) and zeatin produced by Phanerochaete chrysosporium ME446. Bulg. J. Plant Physiol. 1996, 22, 105-110. 